\title{
La cerámica caolín en la cultura Cajamarca (sierra norte del Perú): el caso de la fase Cajamarca Media
}

La céramique au kaolin de la culture Cajamarca, dans les montagnes du nord du Pérou : le cas de la période Cajamarca du milieu

Kaolin ceramics of the Cajamarca culture, northern highlands of Peru: a case study of the Middle Cajamarca phase

\section{Shinya Watanabe}

\section{OpenEdition}

\section{Journals}

Edición electrónica

URL: http://journals.openedition.org/bifea/2592

DOI: $10.4000 /$ bifea.2592

ISSN: 2076-5827

\section{Editor}

Institut Français d'Études Andines

\section{Edición impresa}

Fecha de publicación: 1 agosto 2009

Paginación: 205-236

ISSN: 0303-7495

\section{Referencia electrónica}

Shinya Watanabe, «La cerámica caolín en la cultura Cajamarca (sierra norte del Perú): el caso de la fase Cajamarca Media », Bulletin de I'Institut français d'études andines [En línea], 38 (2) | 2009, Publicado el 01 febrero 2010, consultado el 17 noviembre 2020. URL : http://journals.openedition.org/bifea/2592 ; DOI : https://doi.org/10.4000/bifea.2592

\section{(c) $(1) \odot$}

Les contenus du Bulletin de l'Institut français d'études andines sont mis à disposition selon les termes de la licence Creative Commons Attribution - Pas d'Utilisation Commerciale - Pas de Modification 4.0 International. 


\title{
La cerámica caolín en la cultura Cajamarca (sierra norte del Perú): el caso de la fase Cajamarca Media
}

\author{
Shinya Watanabe*
}

\begin{abstract}
Resumen
Este artículo presenta primero los datos de excavaciones realizadas en el sitio arqueológico Complejo Turístico Baños del Inca —un centro ceremonial ubicado en el departamento de Cajamarca, en la sierra norte del Perú-, y luego considera las características de la cultura Cajamarca en base a la nueva cronología de la cerámica. La cultura Cajamarca se desarrolló después del Periodo Formativo y continuó hasta la llegada de los españoles, manteniendo la misma tradición alfarera de la cerámica caolín. En el valle de Cajamarca se produjo la cerámica caolín del tipo Cajamarca Cursivo Clásico durante la fase Cajamarca Media A (550/600-700 d.C.) y durante la fase Cajamarca Media B y C (700900 d.C.); la cerámica de estilo Cajamarca se distribuyó en una amplia zona que abarcaba la sierra sur y la costa norte. Se piensa que este fenómeno no se debe al intercambio de la cerámica, sino al movimiento de los cajamarquinos y que, a través de su estilo particular, la cerámica cajamarquina habría representado su identidad cultural.
\end{abstract}

Palabras clave: cultura Cajamarca, cerámica caolín, fase Cajamarca Media, identidad cultural

\section{La céramique au kaolin de la culture Cajamarca, dans les montagnes du nord du Pérou : le cas de la période Cajamarca du milieu}

\section{Résumé}

Cet article présente d'abord les données issues des fouilles faites sur le site archéologique «Complejo Turístico Baños del Inca», centre cérémoniel situé dans le département de Cajamarca, région

* Profesor asistente del departamento de Antropología y Filosofía, Nanzan Universidad (Nagoya, Japón). E-mail: shinya@nanzan-u.ac.jp 
montagneuse du Nord du Pérou, puis il précise les caractéristiques de la culture Cajamarca à partir de la nouvelle chronologie de la céramique. La culture Cajamarca s'est dévelopée après la période Formative et a survécu jusqu'à l'arrivée des Espagnols tout en perpétuant la tradition de la céramique au kaolin. La céramique de type Cajamarca Cursive Classique a été produite dans la vallée de Cajamarca durant la période du Cajamarca Moyen A (550/600-700 après J.C.); on la retrouve ensuite dans une zone allant des montagnes du sud à la côte nord du Pérou durant les phases du Cajamarca Moyen $B$ et $C$ (700-900 après J.C.). On s'accorde à penser que ce phénomène n'est pas dû à des échanges de céramique mais à la mobilité des habitants de Cajamarca, et que ce style particulier servaient à renforcer leur identité culturelle.

Mots clés: culture Cajamarca, céramique au kaolin, période Cajamarca du milieu, identité culturelle

\title{
Kaolin ceramics of the Cajamarca culture, northern highlands of Peru: a case study of the Middle Cajamarca phase
}

\begin{abstract}
In this article we begin by presenting the excavation data from the archaeological site Complejo Turìstico Baños del Inca, a ceremonial center, located in the department of Cajamarca in the northern highlands of Peru. After this we offer a consideration of the characteristics of the Cajamarca culture based on a new ceramic chronology. The Cajamarca culture developed after the Formative Period and continued until the arrival of the Spanish, maintaining the same kaolin ceramic tradition. In the Cajamarca basin, the Cajamarca Classic Cursive, a type of the kaolin ceramics, was produced in the Middle Cajamarca Phase A (A.D. 550/600-700) and in the Middle Cajamarca Phase B and C (A.D. 700900). The Cajamarca style ceramics were distributed over a broad zone of the southern highlands and the northern coast. It is thought that this phenomenon did not result from the trade of the ceramics, but from the movement of the Cajamarca people, and that by the particular style of pottery they may have represented their cultural identity.
\end{abstract}

Key words: Cajamarca culture, kaolin ceramic, Middle Cajamarca phase, cultural identity

\section{INTRODUCCIÓN}

Entre las tecnologías inventadas por el hombre, la cerámica tuvo un rol muy importante logrando mejoras en la alimentación. Por ejemplo, al hervir vegetales en agua dentro de recipientes hechos en cerámica, se puede convertir el almidón del tipo ß (beta) en el de tipo $\partial$ (alfa), que es más fácil de digerir. En consecuencia la aparición de la cerámica ha sido generalmente uno de los indicadores que marcan el comienzo de la época Neolítica en el Viejo Mundo.

En las Américas la cerámica apareció por primera vez en la Amazonía alrededor de 5000 a.C. (Roosevelt, 1995) y en Colombia alrededor de 4000 a.C. (OyuelaCaycedo, 1995). En el caso del Perú, la cerámica se introdujo alrededor de 1800 a.C., es decir 2200-3200 años más tarde. Sin embargo, las características de 
la cerámica en los Andes difieren de la cerámica de otras áreas, ya que en el Perú se habían construido centros ceremoniales antes que se introdujera la cerámica. Se piensa que en los Andes la cerámica no se desarrolló solamente para ser usada como utensilio de cocina, sino más bien, como instrumento ritual, por su muy elaborada decoración.

Esta cerámica varía en función de la época y del área; se sabe que la cerámica es un marcador de la unidad cultural, y se supone que la cerámica del mismo estilo traduce un vínculo ritual en el caso del Periodo Formativo y una unidad política o étnica en los periodos posteriores. Son materia de discusión los criterios para correlacionar cultura material y grupos humanos (Reycraft, 2005) y existen múltiples evidencias materiales que pueden ser índice de grupos humanos, siendo una de ellas la cerámica.

En este artículo consideraremos las características culturales andinas en base a la cerámica, tomando como ejemplo la cerámica caolín de la cultura Cajamarca en la sierra norte del Perú. Presentaremos primero las características generales de la cultura Cajamarca y los resultados de las excavaciones en el sitio del Complejo Turístico Baños del Inca. Luego, trataremos algunos aspectos de la cultura Cajamarca y su relación con otras culturas.

\section{LA CULTURA CAJAMARCA Y LA CERÁMICA CAOLÍN}

El valle de Cajamarca se ubica en el lado oriental de la sierra norte del Perú a una altura aproximada de 2 500-2 700 msnm (fig. 1), y es un área muy importante en la arqueología andina ya que se ha registrado en ella una ocupación humana de muy larga duración. Hasta el presente se han encontrado varios centros ceremoniales del Periodo Formativo (1500-50 a.C.) como Huacaloma y Chondorko (fig. 2), pero no se han encontrado centros ceremoniales de la época precerámica (Terada \& Onuki, 1982; 1985). El periodo Formativo se divide en 4 fases: la fase Huacaloma Temprana (1500-1000 a.C.), la fase Huacaloma Tardía (1000-500 a.C.), la fase EL (Early Layzón: 500-250 a.C.) y la fase Layzón (250-50 a.C.). En la fase Layzón, correspondiente a la última fase del Periodo Formativo, se construyeron centros ceremoniales como los sitios de Layzón y Cerro El Consejo (fig. 2), mientras que en otras áreas durante esta época se dejó de edificarlos.

La cultura Cajamarca (50 a.C.-1532 d.C.) se desarrolló después de la fase Layzón, y se caracterizó por la producción de la cerámica en caolín. Los índices que separan la fase Layzón y la cultura Cajamarca son, entre otros, la aparición de la cerámica caolín, los cambios ocurridos en la composición de la misma y la mampostería de la arquitectura. En el periodo Formativo toda la cerámica se usaba en los centros ceremoniales para la actividad ritual, en consecuencia resulta difícil distinguir la cerámica fina (diagnóstica) y la cerámica tosca (no-diagnóstica). Sin embargo, en el periodo de la cultura Cajamarca se nota la diferencia entre la cerámica caolín y la no caolín; esta división corresponde aproximadamente a las cerámicas fina y tosca y se podría decir que en la cultura Cajamarca existe una marcada diferenciación de las funciones de la cerámica. La cultura Cajamarca se 


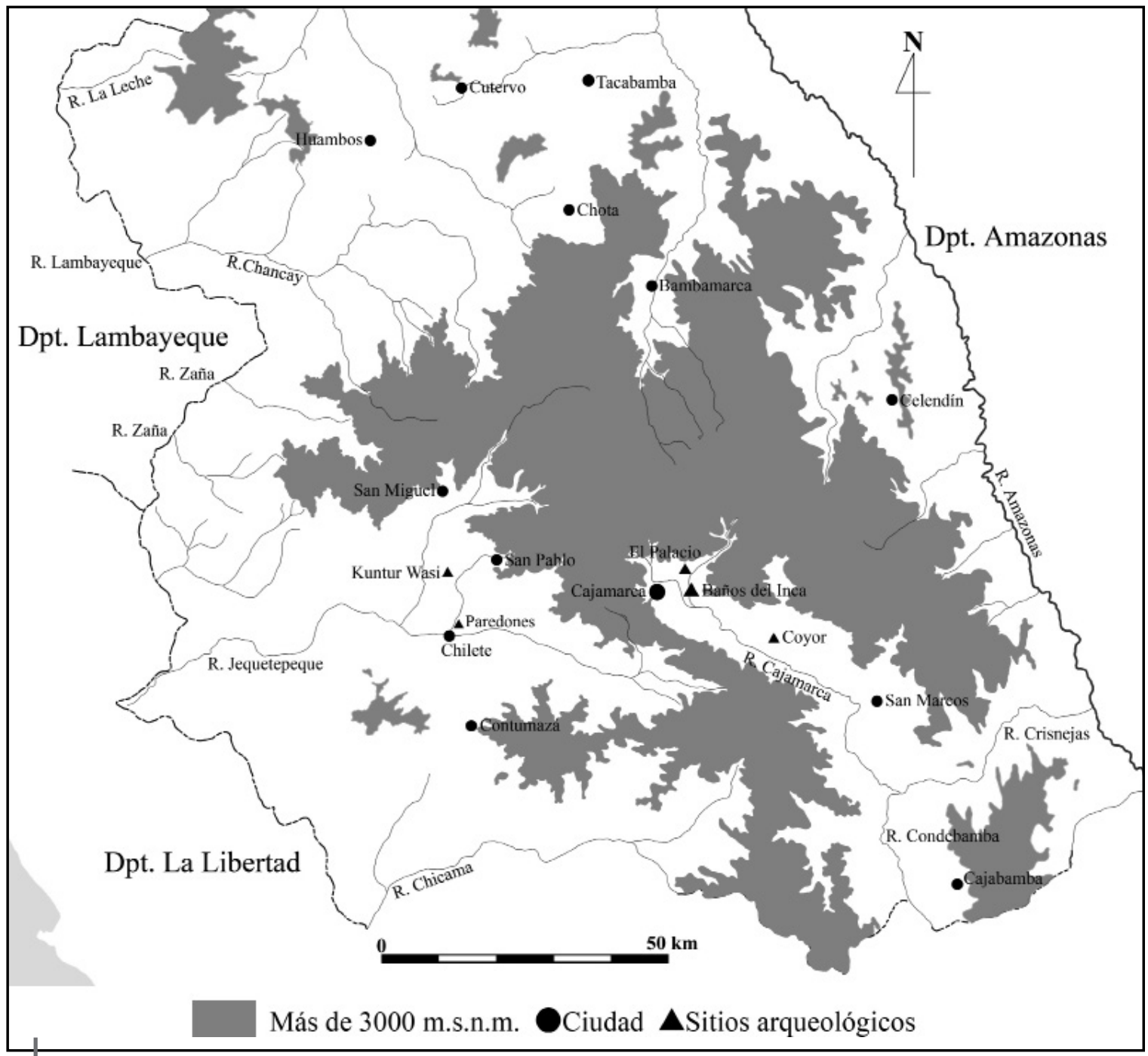

Figura 1 - Mapa del departamento de Cajamarca 


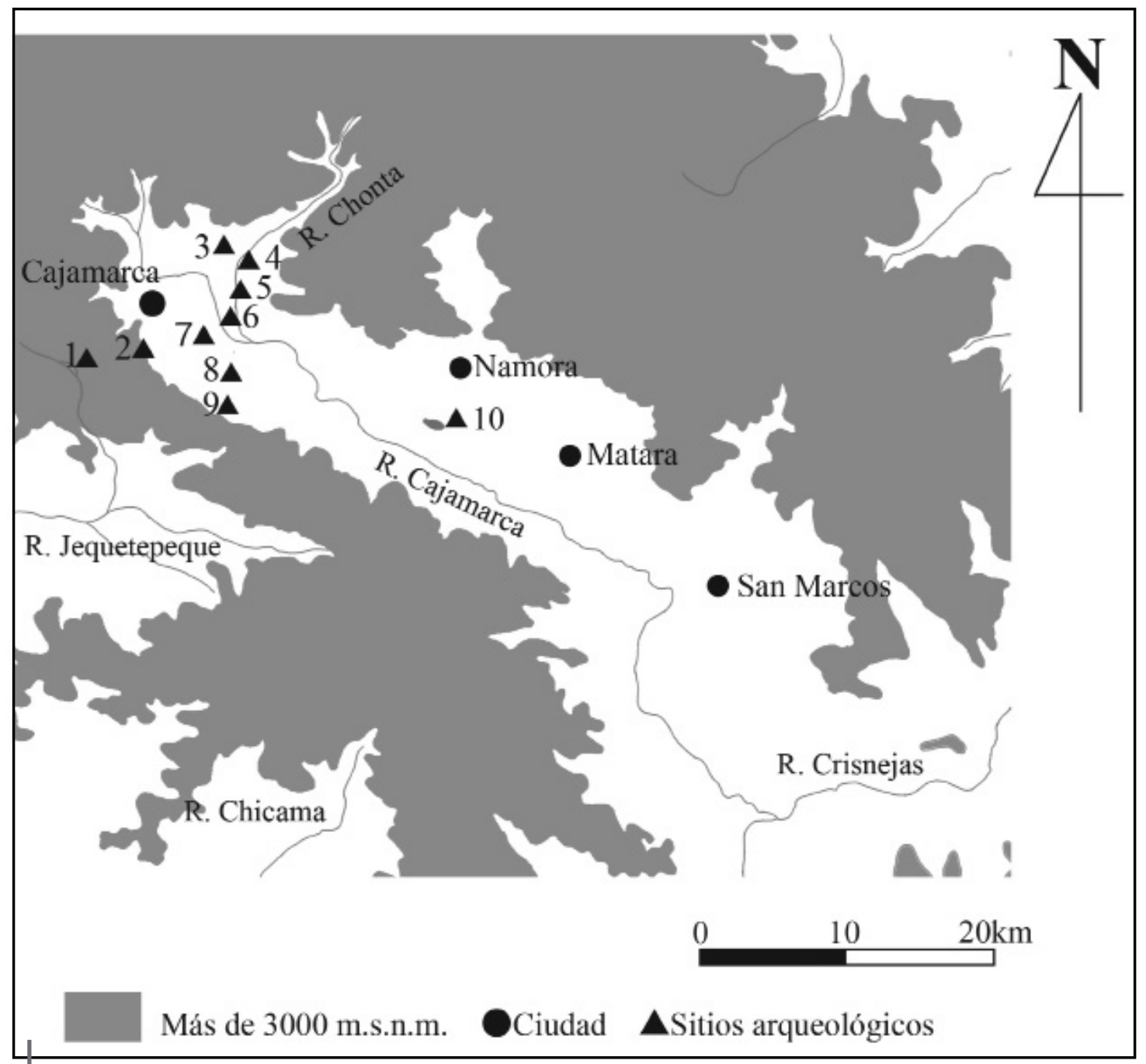

Figura 2 - Mapa de la ubicación de los sitios arqueológicos en el valle de Cajamarca

1: El Consejo, 2: Layzón, 3: El Palacio; Kolguitín, 4: Chondorco, 5: Complejo Turístico Baños del Inca, 6: Wairapongo, 7: Huacaloma, 8: Huacariz, 9: Amoshulca, 10: Coyor 
define por la presencia de la cerámica caolín, la cual presenta cambios notables según sus fases en el dibujo, la pasta y la forma, haciendo fácil su identificación en otras áreas (Schjellerup, 1997: 208). Por otro lado, no se sabe mucho sobre otros aspectos culturales de la cultura Cajamarca.

La palabra «caolín» proviene del pueblo Gaoling en China, que es la región productora de arcilla para Jing de Zhen, un centro famoso de alfarería. Caolín es el nombre de un grupo de arcilla que se compone de los minerales caolín: caolinita, nacrita, dikita, haloisita, etc. La fórmula química de caolinita, nacrita y dikita es $\mathrm{Al}_{4} \mathrm{Si}_{4} \mathrm{O}_{10}(\mathrm{OH})_{8}$ y la de la haloisita es $\mathrm{Al}_{4} \mathrm{Si}_{4} \mathrm{O}_{10}(\mathrm{OH}) \bullet 4 \mathrm{H}_{2} \mathrm{O}$. El caolín es el producto de alteración hidrotermal del feldespato, y su causa principal de formación es la alteración hidrotermal de la roca volcánica. Por otro lado, existe el caolín sedimentario o de erosión eólica (eflorescente). El caolín tiene refractaridad y puede fabricar cerámica de color blanco sin deformarse, pero no tiene plasticidad por lo que no puede usarse como arcilla, necesitando ser mezclado con otros minerales como temperante.

Un análisis de la pasta de la cerámica caolín fue efectuado recientemente por Masaaki Shimizu y Yuji Seki. El estudio ha permitido definir por lo menos 3 canteras de caolín1 en el valle de Cajamarca (Seki et al., 2001; Seki \& Ugaz, 2002; Seki \& Tejada, 2003).

La mayoría de la cerámica caolín Cajamarca presenta la forma de taza con base anular o redonda; no se fabricaron botellas, cántaros, escudillas o tinajas. La decoración es pictórica con diseños geométricos y naturales representando a felinos y a humanos, escaseando la decoración plástica. Se usaron los colores como el negro, rojo, blanco y morado, siendo este último usado en menor cantidad. También existe la cerámica caolín no decorada.

Una característica de la cultura Cajamarca es la fabricación de cucharas elaboradas en caolín2 (figs. 3-4). Por algunas razones se podría interpretar que había intención de usar la taza de caolín con la cuchara, a pesar de que el porcentaje de las cucharas es bajo en relación con el de las tazas. En primer lugar, la taza y la cuchara comparten los mismos diseños; en segundo lugar, la forma de la cerámica caolín es mayormente una taza con boca abierta, no muy profunda, permitiendo el alcance de la cuchara. Por otro lado, en la cerámica caolín no se han encontrado botellas, cántaros o vasijas muy profundos.

No tenemos evidencias sobre la actividad realizada con la cerámica caolín y la cuchara. Parece que no se usaban para las comidas cotidianas, sino para una actividad ritual porque presentan diseños muy elaborados tanto en el lado exterior como en el interior de la taza. Una posibilidad es que las cucharas fueran utilizadas como parte de la parafernalia ritual; preo, para confirmar esta hipótesis necesitamos mayores datos que indiquen la verdadera función de dicho instrumento.

1 Dos de ellas, Mollepampa y Shudal, están ubicadas cerca de la ciudad de Cajamarca y la tercera, se encuentra próxima al distrito de Namora.

2 Podemos considerar cierta continuidad dentro del valle de Cajamarca, pues existen cucharas de cerámica desde el Periodo Formativo. 


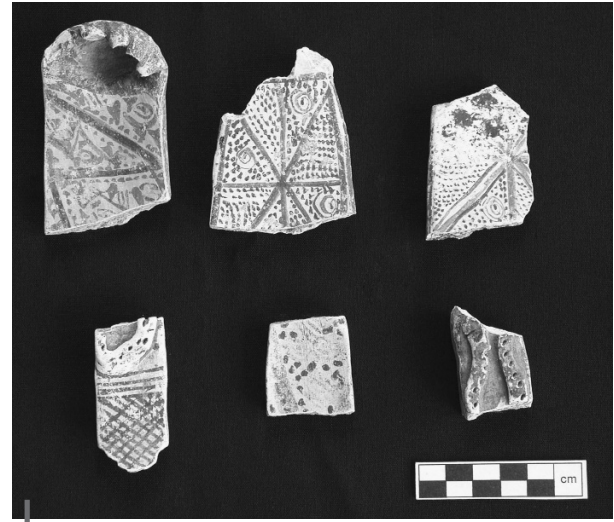

Figura 3 - Mangos de cuchara recuperados en el Complejo Turístico Baños del Inca

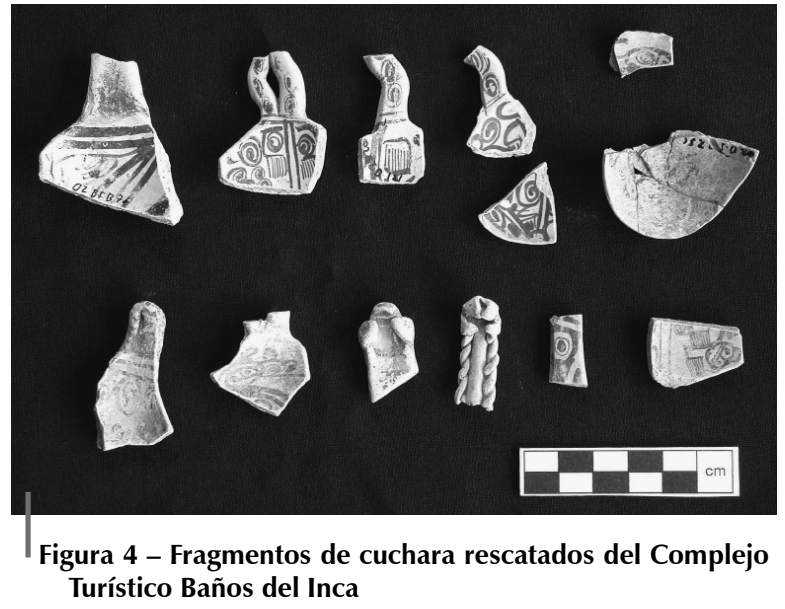

Turístico Baños del Inca

En el valle de Cajamarca es muy fácil encontrar fragmentos de caolín de la cultura Cajamarca en la superficie de los sitios arqueológicos. Normalmente se supone que la cerámica fina se asocia a un contexto especial, como por ejemplo a una tumba o a una ofrenda, pero podemos decir que la cerámica caolín es abundante en el contexto doméstico y no se limita solamente al contexto funerario, aunque poco sabemos sobre el patrón funerario de la cultura Cajamarca.

Son notables las características de la cerámica caolín de la cultura Cajamarca si las comparamos con las de la cultura Recuay (Grieder, 1978; Lau, 2006). En la cultura Recuay la mayoría de la cerámica caolín se ha encontrado hasta ahora en contextos funerarios, y es muy raro hallarla en contextos domésticos. Además existe la vasija de caolín, como el cántaro, que no fue usada junto a la cuchara, así que podemos decir que el significado cultural de la cerámica caolín es diferente entre las dos culturas.

En el valle de Cajamarca se distribuye la cerámica caolín con las mismas características tanto en la forma como en el dibujo, haciendo difícil especificar el centro de producción o distribución de la cerámica. Tampoco hemos encontrado una concentración de cerámica caolín dentro de un mismo sitio; se distribuye en todas partes, y no se nota diferencia en su calidad o abundancia.

En la producción de la cerámica caolín, se observa una tendencia que no se orienta hacia una diferenciación entre ellas, sino hacia una uniformidad, ya que existe un determinado repertorio de diseño, se usa arcilla de la misma calidad y se aplica el engobe para que tenga el mismo color. Se nota una orientación clara hacia la homogeneidad. La característica de la cerámica caolín concuerda con el patrón de asentamiento. Es difícil especificar el centro político que podría considerarse como la «capital», y se supone que en el valle de Cajamarca no existía una sociedad estratificada verticalmente, sino más bien un conjunto de sociedades que tuvieron un vínculo horizontal. Si la cerámica caolín representa una relación ritual compartida entre ellas, se podría decir que la cultura Cajamarca se define por la misma costumbre ritual. 
La cerámica es uno de los índices de desarrollo cultural y en los Andes prehispánicos varias culturas elaboraron la suya propia, siendo la cultura Cajamarca una de ellas. Sin embargo, la carencia de cerámica de buena calidad no significa necesariamente una falta de desarrollo cultural. Por ejemplo, en la zona de Huamachuco, al sur del valle de Cajamarca, no hubo mucho interés por la cerámica (Lange Topic \& Topic, 1987: 20), empero existió una tradición arquitectónica muy desarrollada (Lange Topic \& Topic, 1987: 1; Topic \& Lange Topic, 2001: 183, 187).

\section{LA CRONOLOGÍA EN EL VALLE DE CAJAMARCA Y LA FASE CAJAMARCA MEDIA}

Los pioneros en realizar investigaciones arqueológicas en forma sistemática en el valle de Cajamarca fueron los franceses Henry \& Paule Reichlen (Reichlen \& Reichlen, 1949). Ellos registraron 93 sitios y excavaron 5 de ellos, proponiendo la primera cronología para el valle de Cajamarca que se compone de 6 periodos: el periodo Torrecitas-Chavín, Cajamarca I, II, III, IV y V.

Luego, desde el año 1979 la Expedición Científica Japonesa a la cuna de América dirigida por Kazuo Terada ha realizado excavaciones arqueológicas en el valle de Cajamarca, siendo su objetivo principal esclarecer el proceso de desarrollo de las sociedades del periodo Formativo. Se realizaron excavaciones en Huacaloma, Layzón, Kolguitín, Huacariz, Wairapongo y Amoshulca (fig. 2), y se presentó una nueva cronología de la cultura Cajamarca, hecha de 5 fases: la fase Cajamarca Inicial (50 a.C.3-200 d.C.; se divide en 2 subfases A y B), la fase Cajamarca Temprana (200-450 d.C.; se divide en 3 subfases A, B y C), la fase Cajamarca Media (450-900 d.C.; se divide en 2 subfases A y B), la fase Cajamarca Tardía (900-1200 d.C.) y la fase Cajamarca Final (1200-1532 d.C.) (Matsumoto, 1993; Terada \& Matsumoto, 1985; Terada \& Onuki, 1982; 1985).

Ambas cronologías se basan en el análisis de la cerámica caolín, pero la cerámica de otro tipo no ha sido clasificada sistemáticamente 4 y no se sabe mucho sobre la arquitectura y el patrón funerario de esta cultura. Analicemos ahora las características de la cultura Cajamarca tomando como ejemplo el caso de la fase Cajamarca Media. En esta fase se puede observar la integración de las tradiciones de cerámica en comparación con la fase anterior: la pasta es sumamente compacta y el diseño muy minucioso.

3 La fecha absoluta le pertenece a Matsumoto (1993), pero adopta la fecha 200 a.C. para el comienzo de la fase Cajamarca Inicial. En este artículo se emplea 50 a. C. para el inicio de la fase Cajamarca Inicial, suponiendo la contemporaneidad de la fase Layzón y la fase Sotera del sitio Kuntur Wasi (Onuki, 1995). Además, como se discute más adelante, el autor escoge la fecha 550/600 d.C. para el principio de la fase Cajamarca Media, en lugar de 450 d.C.

4 Se ha clasificado la cerámica de otro tipo, recuperada en Huacaloma en 1979, en las fases Cajamarca Inicial, Temprana y Media (Terada \& Onuki, 1982). 
A pesar de que hay uniformidad en la cerámica, no está claro el patrón de asentamiento; no se sabe por ejemplo si existió un centro, y si existió, dónde estuvo situado ese centro. Terada \& Ryozo Matsumoto mencionan al lugar de Coyor (fig. 2) como un centro político de esta fase y también Daniel G. Julien (1988) es de la misma opinión. Hasta hace poco no teníamos muchos datos de excavaciones de dicha fase, pero se obtuvieron datos nuevos en las excavaciones arqueológicas realizadas en el Complejo Turístico Baños del Inca.

\section{EXCAVACIONES EN EL COMPLEJO TURÍSTICO BAÑOS DEL INCA}

Baños del Inca se encuentra ubicado en la margen izquierda del río Chonta, afluente del río Cajamarca, a cinco kilómetros al este de la ciudad de Cajamarca (figs. 1-2). Hoy en día se encuentra allí el balneario público llamado Complejo Turístico Baños del Inca. Tenemos al respecto la información proveniente de las investigaciones arqueológicas realizadas por el Instituto Nacional de CulturaCajamarca bajo la conducción de Rogger Ravines quien, en el año 1979, escribió lo siguiente:

«Trabajos de delimitación arqueológica realizados por el Instituto Nacional de Cultura, en septiembre de 1979, pusieron al descubierto numerosos restos arquitectónicos de diversa índole, inclusive canales y estanques que concuerdan con los datos y descripciones de los primeros conquistadores españoles» (Ravines, 1985: 110).

Para definir a qué fase pertenece este sitio, realizamos excavaciones durante 4 semanas en el año 2001 y 8 semanas en el 2002; (Watanabe \& Ugaz, 2002; Watanabe et al., 2003). Según los resultados ha quedado aclarado que el sitio no corresponde al periodo incaico, sino que se construyó y utilizó desde fines de la fase Cajamarca Temprana hasta la primera mitad de la fase Cajamarca Media. La arquitectura se asocia a la cerámica caolín que corresponde al tipo Cajamarca Precursivo de la fase Cajamarca Temprana C (fig. 5) y al Cajamarca Cursivo Clásico de la fase Cajamarca Media A (figs. 6-7). Se recuperaron fragmentos de cucharas (figs. 3-4), platos redondos de base plana y fragmentos de antara hecha en arcilla.

El terreno en donde realizamos las excavaciones presentaba una ligera pendiente con dirección de este a oeste, es decir hacia el río Chonta; se confirmó que este fue nivelado varias veces y rellenado con bastante tierra en la parte oeste.

A pesar de que encontramos solamente las bases de los muros, hemos logrado reconstruir en forma general el diseño arquitectónico del sitio, el mismo que presenta una estructura con una plaza (fig. 8); esto quiere decir que dicho sitio no habría sido una vivienda cotidiana, sino un centro ceremonial. En el sitio existe una plaza de $16 \times 13$ m, alrededor de la cual se ubican las plataformas y los recintos. Los accesos se encuentran en cada esquina de la plaza, a pesar de que no hemos confirmado la esquina norte. El acceso sur tiene 1,3-1,5 m de ancho, y se conecta con el espacio más amplio del sur. La Escalera C1, ubicada en la esquina 

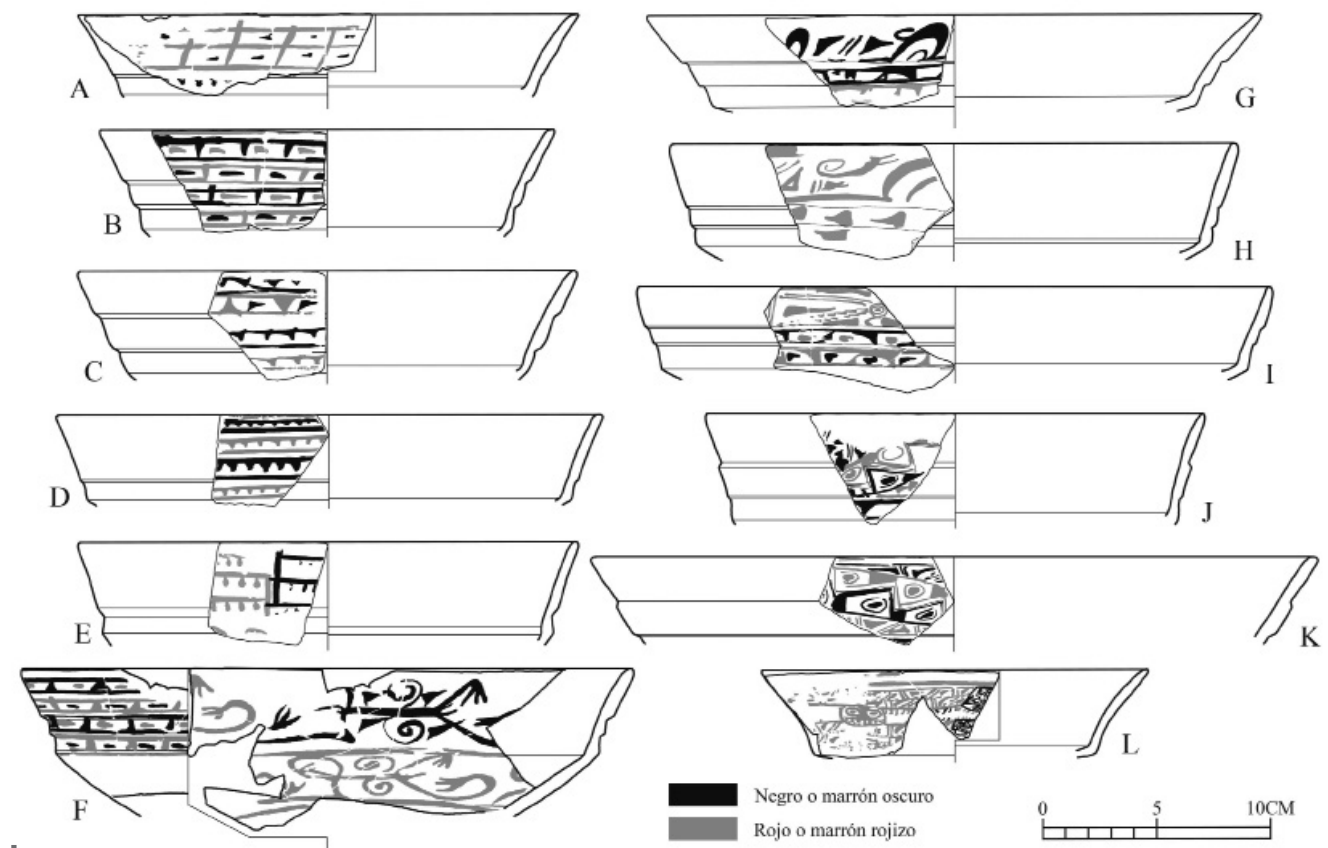

Negro o marrón oscuro

Rojo o marrón rojizo

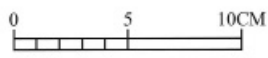

Figura 5 - Cajamarca Precursivo

Complejo Turístico Baños del Inca
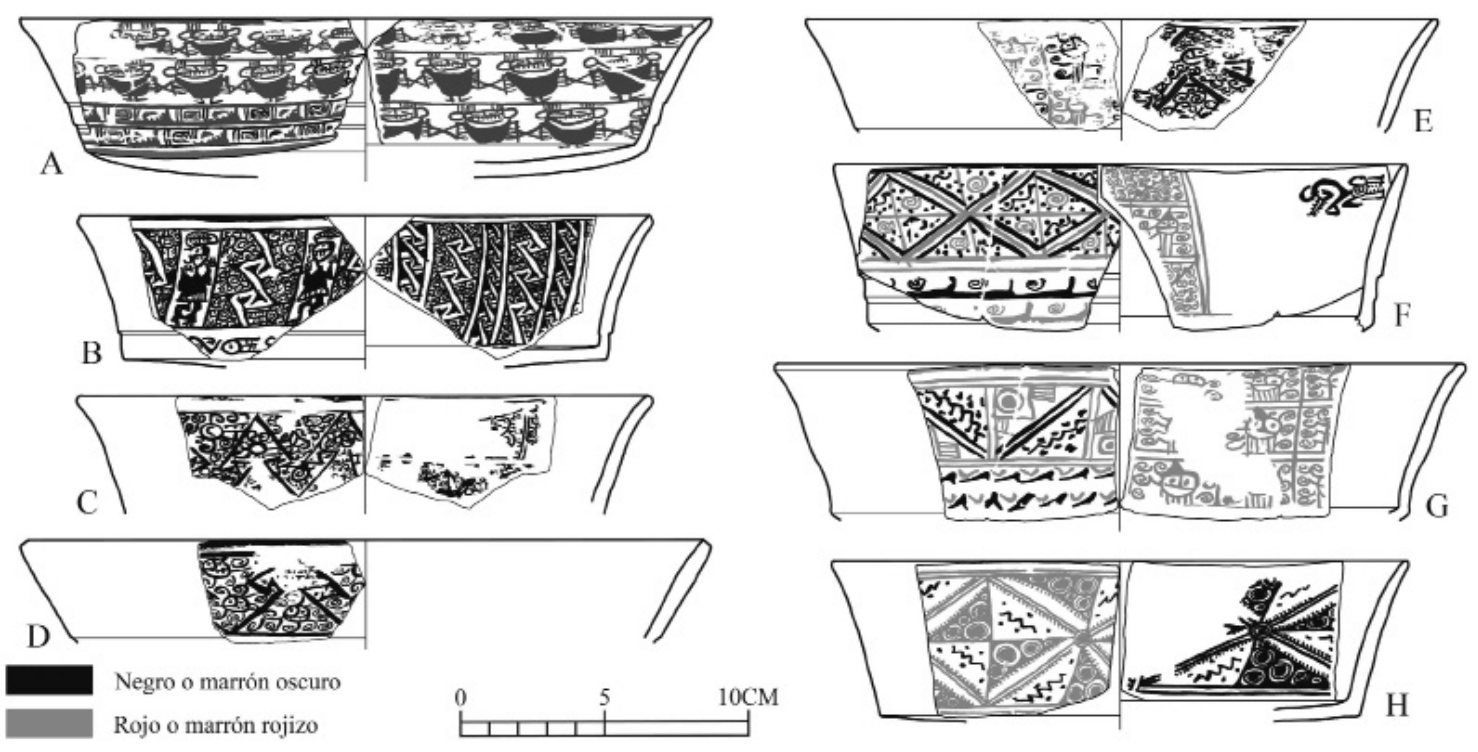

Figura 6 - Cajamarca Cursivo Clásico

Complejo Turístico Baños del Inca

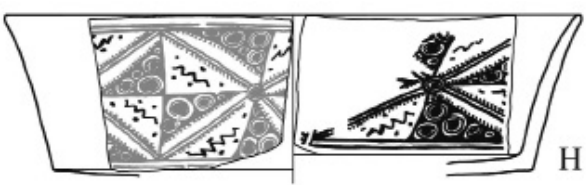



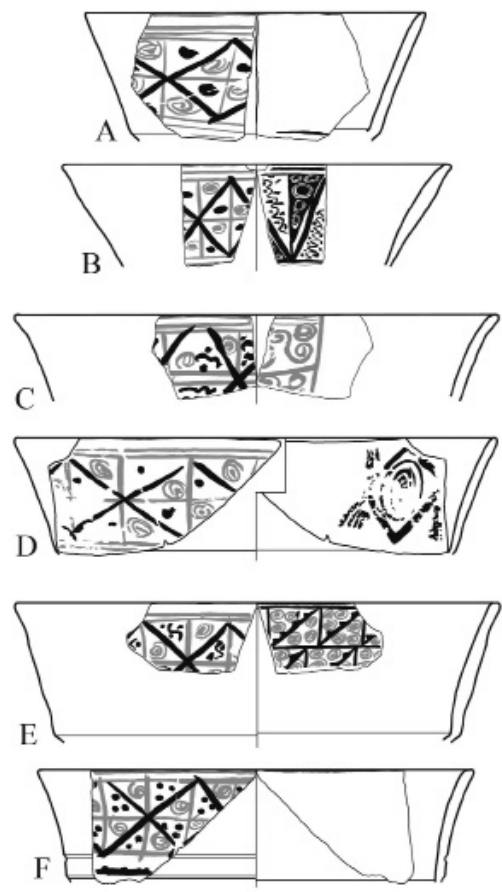
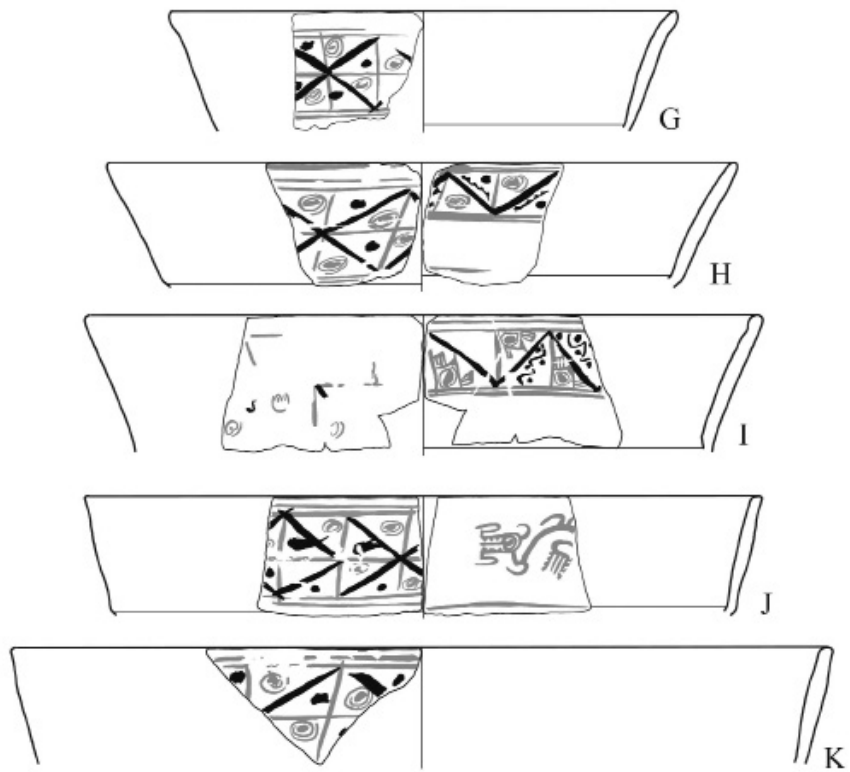

Negro o marrón oscuro

Rojo o marrón rojizo

Figura 7 - Cajamarca Cursivo Clásico con el motivo de radio Complejo Turístico Baños del Inca

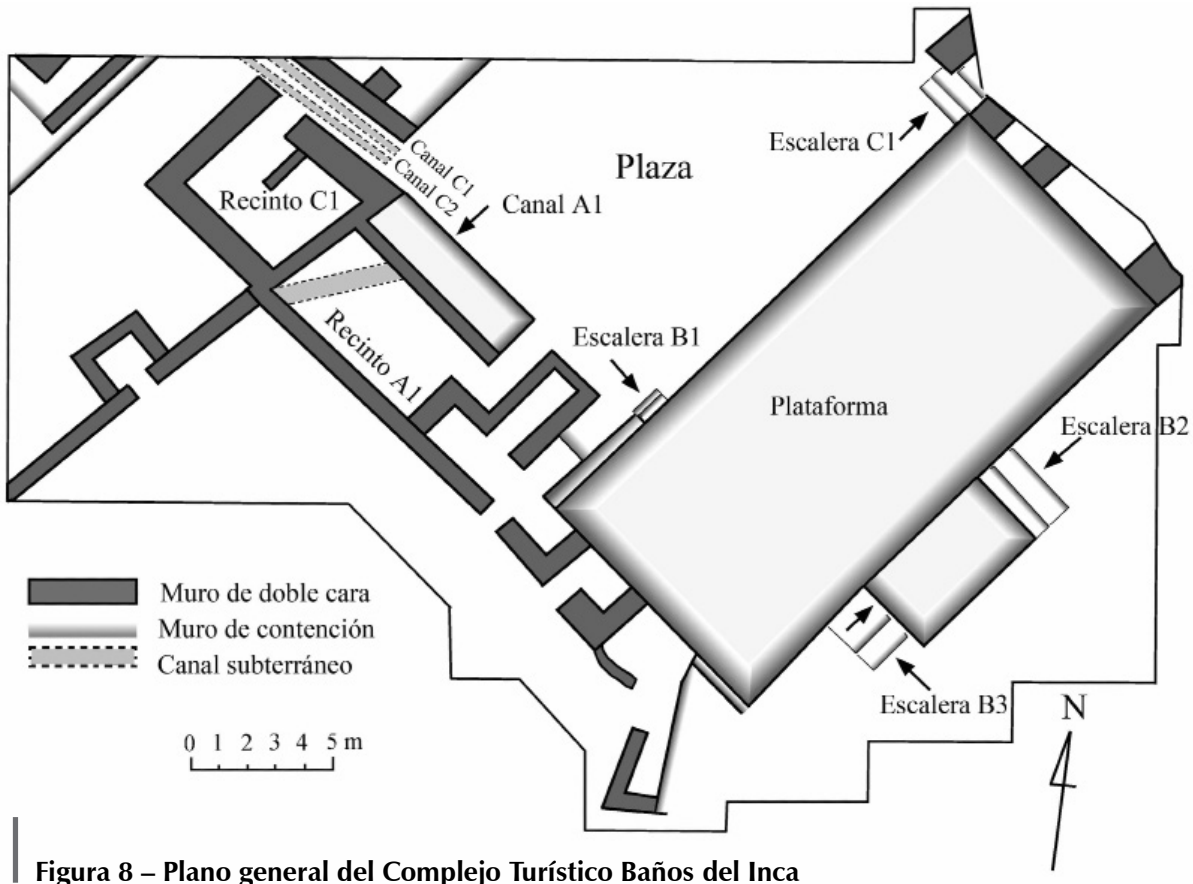


este, accedería a la arquitectura más grande. Por lo tanto, se puede decir que la arquitectura encontrada constituye parte de un gran conjunto arquitectónico que no hemos podido definir en su totalidad, debido a la alteración causada por la presencia de edificios modernos.

Al sureste de la plaza se ubica una gran plataforma de 20,6 x 9,5 m (fig. 9), cuya altura no pudimos confirmar puesto que la parte superior ha sido destruida. Se ha confirmado que el interior de la plataforma fue dividido en pequeños espacios, los mismos que fueron rellenados con piedra y tierra. Esta técnica constructiva es conocida como «encajonamiento» y sirve para dispersar la presión y darle estabilidad a la plataforma5. Desde la plaza se accede a la plataforma por la Escalera B1. A la plataforma grande se adosa una pequeña de 5,4 x $3 \mathrm{~m}$, con la Escalera B2 en el lado noreste y la Escalera B3 en el lado suroeste. También existieron más recintos pequeños alrededor de la plataforma.

Al interior de la plataforma grande encontramos un recinto más antiguo, el cual tiene la misma orientación que la plataforma y mide 10,5 m (el eje suroestenoreste) por más de $7 \mathrm{~m}$ (el eje noroeste-sureste), aunque no pudimos encontrar el muro oeste pues había sido derribado (fig. 9). Los muros tienen $60 \mathrm{~cm}$ de ancho

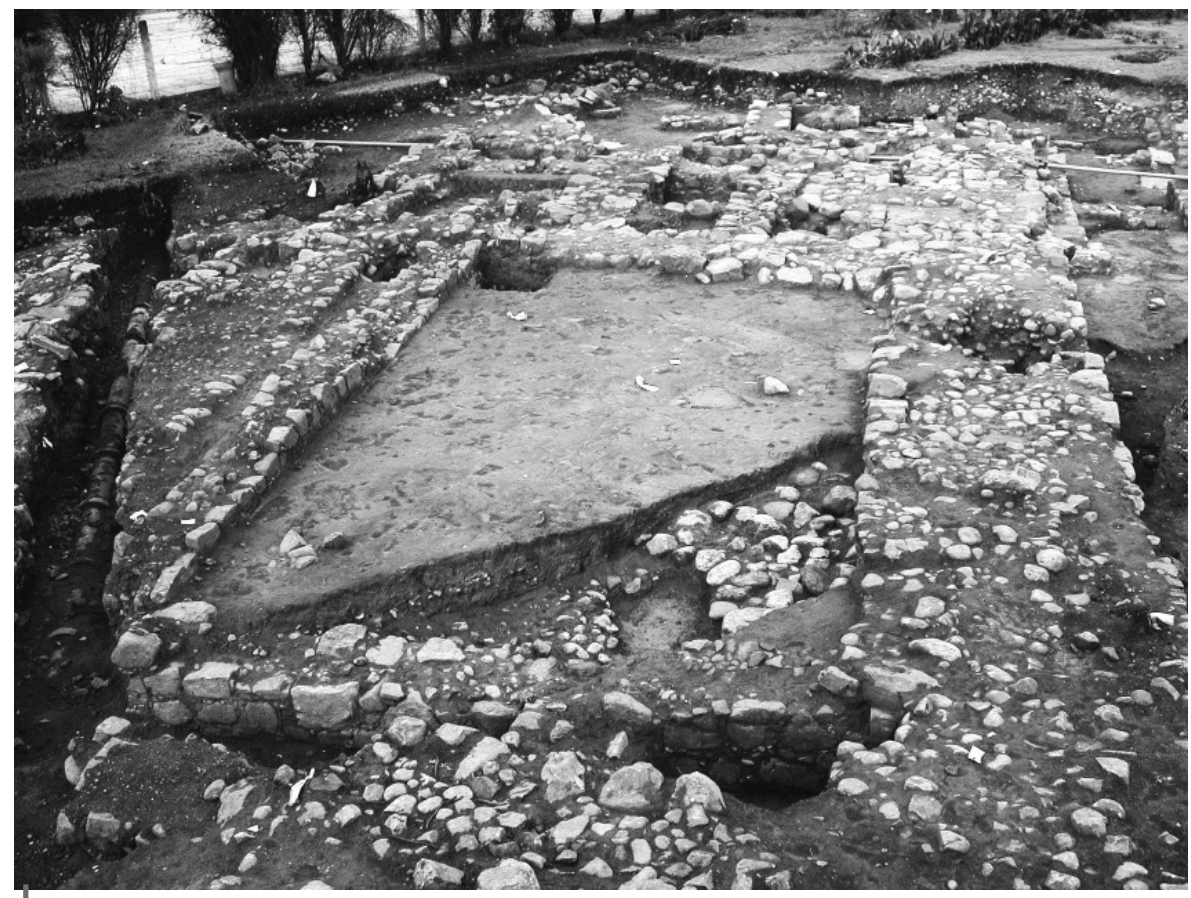

Figura 9 - Plataforma grande vista desde el noreste

Complejo Turístico Baños del Inca

5 Se llama chamber-and-fill technique en inglés. En la costa norte aparece en Moche $\mathrm{V}$ y parece haber sido introducido desde la costa central (Shimada, 1994: 160). 
y el lado interior del recinto es $60 \mathrm{~cm}$ más alto que su lado exterior. Este recinto está asociado con la cerámica caolín del tipo Cajamarca Precursivo de la última subfase de la fase Cajamarca Temprana, y no hemos encontrado otra arquitectura que perteneciera a esta fase.

El Recinto A1, ubicado al suroeste de la plaza, tiene una dimensión de 3,4 × 6,4 $\mathrm{m}$ y en el lado noreste se adosa una terraza pequeña con una altura de $30 \mathrm{~cm}$. Asimismo encontramos el Canal A1 en el lado noreste que pasa por debajo del piso del Recinto A1, pero no continúa más, así que pensamos que no fue terminado.

El Recinto $\mathrm{C} 1$, adosado al lado noroeste del Recinto A1, tiene una dimensión de 3,5 x 4,5 m; debajo del piso del pasadizo del lado noreste están ubicados 2 canales. El Canal C2 está destruido por el Canal C1, pero no sabemos si eso significa una diferencia temporal o un proceso constructivo.

No deja de ser interesante la condición del abandono del sitio. Sobre el piso, alrededor de la plataforma grande, estaba acumulada tierra quemada de $1-2 \mathrm{~cm}$ de espesor, alcanzando hasta $25 \mathrm{~cm}$ dentro de un recinto al suroeste de la plataforma. Además encontramos muchos fragmentos de revoque con las huellas de caña, los cuales pertenecerían a los muros de los edificios que habrían existido encima de la plataforma o a su alrededor. Sobre la tierra quemada estaba acumulada tierra amarilla compacta con piedras grandes con diámetros de 30-40 cm; estas piedras habrían constituido parte de los muros de la arquitectura porque presentan las caras trabajadas. Además se ha observado la destrucción intensa de las esquinas de los edificios.

Encima de la esquina norte del Recinto $\mathrm{C} 1$, se encontró un contexto funerario. El individuo se encontraba en posición extendida con la cabeza hacia el este, pero no tenía ninguna ofrenda. Posiblemente se trate de un sacrificio ritual asociado al abandono del sitio. En resumen, en un momento el sitio fue incendiado intencionalmente, derribándose los edificios, para luego ser cubierto totalmente, convirtiéndose en un terreno plano como si no hubiera existido nada. No sabemos si esto fue obra de la misma gente que había utilizado el sitio o de foráneos. Más adelante volveremos sobre las causas del abandono.

Los materiales carbonizados recuperados sobre el piso presentan fechas de C14 de $1325 \pm 30$ (TKa-13726), $1480 \pm 30$ (TKa-13727), $1355 \pm 30$ (TKa-13728); calibrados por el programa OxCal v. 4.0.1 las fechas del abandono se ubicarían alrededor de 700 d.C. (cuadro 1). La segunda muestra (TKa-13727) es más antigua que las otras dos, y parece ser de material reutilizado como madera o una inclusión. 
Cuadro 1 - Fechas de C14, calibrado por OxCal v.4.0.1 (Bronk Ramsey, 1995; 2001; 2006), en base a SHCal04 (MaCormac et al., 2004)

\begin{tabular}{|c|c|c|c|c|}
\hline $\mathbf{N}^{0}$ & 14C a.p. & $\delta 13 C$ & Cal d.C. $(1 \sigma)$ & Cal d.C. $(2 \sigma)$ \\
\hline TKa-13726 & $1325 \pm 30$ & $-22,1$ & $689-772$ d.C. $(68,2 \%)$ & $\begin{array}{l}664-822 \text { d.C. }(93,1 \%) \\
842-860 \text { d.C. }(2,3 \%)\end{array}$ \\
\hline ТКа-13727 & $1480 \pm 30$ & $-21,4$ & 607-651 d.C. (68,2 \%) & 572-661 d.C. $(95,4 \%)$ \\
\hline ТКа-13728 & $1355 \pm 30$ & $-21,5$ & 668-715 d.C. (47,5 \%) & $\begin{array}{l}659-775 \text { d.C. }(95,4 \%) \\
745-768 \text { d.C. }(22,5 \%)\end{array}$ \\
\hline
\end{tabular}

\section{MODIFICACIÓN DE LA CRONOLOGÍA DE LAS FASES CAJAMARCA MEDIA Y TEMPRANA}

\section{1. La fase Cajamarca Media}

La fase Cajamarca Media corresponde al Periodo Cajamarca III de la cronología de los Reichlen. Según ellos se fabricaron 2 tipos de cerámica cursiva, que aparecen en forma secuencial y temporal; estos fueron definidos como Cursivo Clásico y Cursivo Floral (figs. 6, 7, 10). Sin embargo, Terada \& Matsumoto no están de acuerdo con esta propuesta y sentencian así:

«Después que los Reichlen sugirieron que el estilo Cursivo Floral sucedió al estilo Cursivo Clásico, muchos estudiosos han usado dichos estilos como indicadores eficientes para subdividir la época Cajamarca III. Sin embargo, nuestro material señala que la situación es más complicada. El estilo Cursivo Clásico existe solamente en la primera mitad, pero el cursivo floral, que abunda en la segunda mitad, se halla también en la primera. Por lo tanto, no podemos decir que el cursivo floral sea característico de la segunda mitad (...) Hecho importante es que el tipo Cajamarca Cursivo Floral es dominante en la Subfase A» (Terada \& Matsumoto, 1985: 81; el énfasis es nuestro).

Además Terada y Matsumoto establecieron un nuevo tipo de cerámica caolín Cajamarca Cursivo Rectilíneo, separándolo del Cajamarca Cursivo Clásico y presentaron una nueva cronología de la fase Cajamarca Media, según la cual en la subfase Cajamarca Media A se producen los 3 tipos de la cerámica cursiva, Cajamarca Cursivo Clásico, Cajamarca Cursivo Rectilíneo y Cajamarca Cursivo Floral y en la subfase Cajamarca Media B se producía solamente Cajamarca Cursivo Floral, cuya pasta y dibujos se volvieron más toscos, y aparecieron por primera vez en la cerámica caolín los trípodes cortos.

En las investigaciones realizadas en el Complejo Turístico Baños del Inca pudimos obtener los datos necesarios para resolver esta contradicción en la cronología de la fase Cajamarca Media. Se han recuperado cerca de 39600 tiestos de cerámica prehispánica y moderna, de los cuales unos 5600 son parte de bordes. Se han clasificado todos los fragmentos de borde, de los cuales cerca de $40 \%$ han sido de caolín. Uno de los resultados importantes nos indica que existió un tiempo 
en el cual se producía solamente el Cajamarca Cursivo Clásico sin presencia del Cajamarca Cursivo Floral. Vale enfatizar que en todas las áreas excavadas no se ha encontrado ni un solo fragmento del Cajamarca Cursivo Floral asociado a la arquitectura. Solo se han recuperado 2 fragmentos del Cajamarca Cursivo Floral junto con los fragmentos coloniales o modernos en la capa superior, que seguramente fueron trasladados para nivelar la parte oeste del sitio; por ello estos 2 fragmentos no se pueden asociar con la arquitectura.
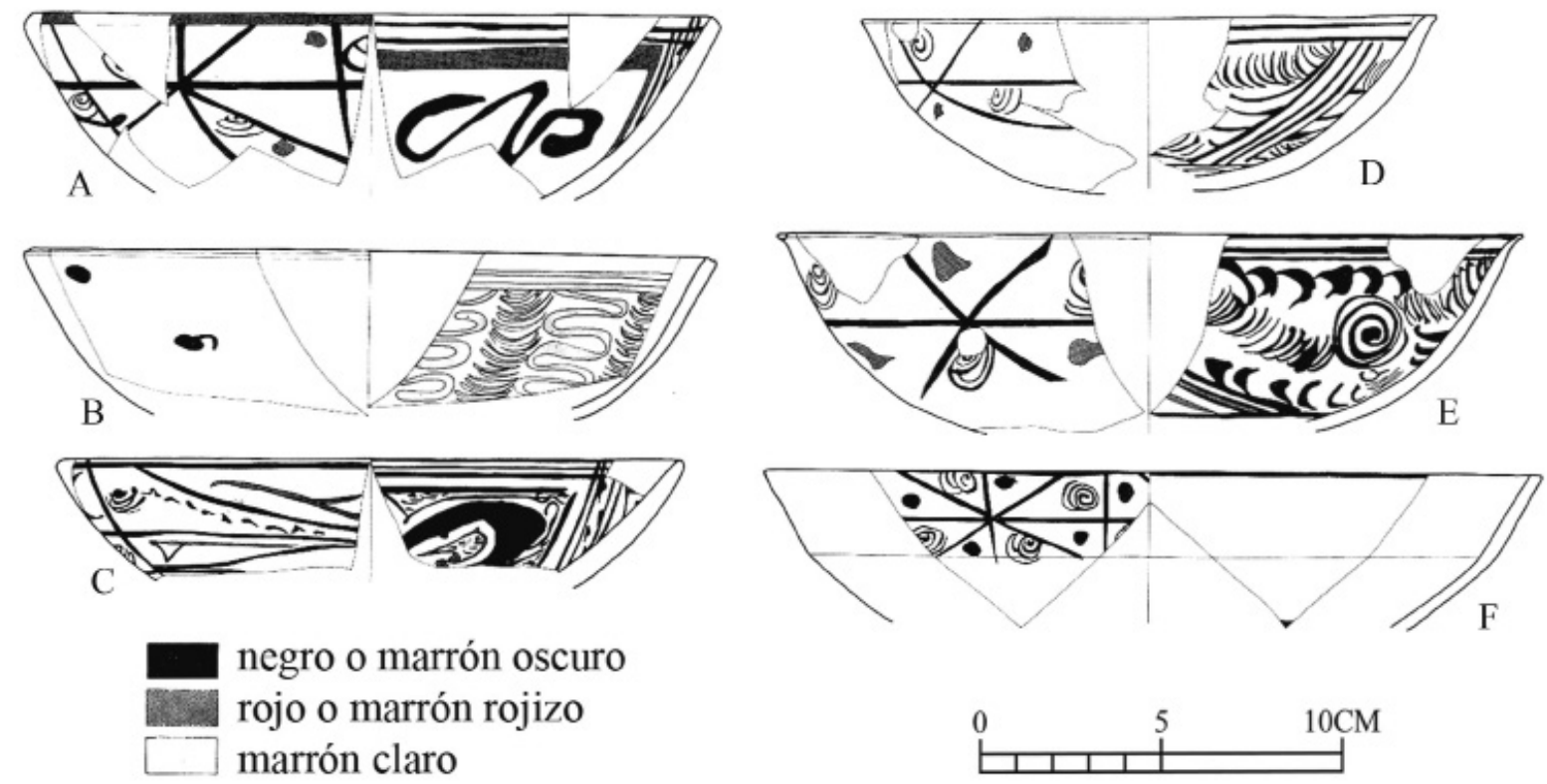

Figura 10 - Cajamarca Cursivo Floral

Fuente: Terada \& Onuki, 1982: PLATE 104

Además encontramos en el mismo estrato cerámica Cajamarca Precursivo de la fase Cajamarca Temprana y cerámica Cajamarca Cursivo Clásico. Nosotros interpretamos que se incluyó el Cajamarca Precursivo en el estrato de Cajamarca Cursivo Clásico, o que hubo un corto tiempo transitorio durante el cual se producían ambos tipos. De todas maneras podemos decir que el Cajamarca Precursivo y el Cajamarca Cursivo Clásico aparecen sucesivamente. Los 2 tienen presencia en el mismo estrato, pero eso no significa necesariamente que estos 2 sean contemporáneos o que comenzaron a producirse al mismo tiempo.

Terada y Matsumoto manifiestan que el Cajamarca Cursivo Floral se halla desde el comienzo de la fase Cajamarca Media, indicando que se han recuperado en los mismos estratos Cajamarca Cursivo Clásico junto con Cajamarca Cursivo Floral. Este dato significaría que los 2 tipos de cerámica cursiva fueron producidos 
sucesivamente y en el estrato de Cajamarca Cursivo Floral se incluyó Cajamarca Cursivo Clásico del tiempo anterior, pero esto no significa que los 2 empezaron a producirse al mismo tiempo.

Por lo tanto, se puede decir que Cajamarca Cursivo Clásico apareció antes que Cajamarca Cursivo Floral. Además, si es válida la observación de Terada y Matsumoto de que en la época posterior Cajamarca Cursivo Floral se volvió más tosco y aparece con trípodes, entonces se podría dividir la fase Cajamarca Media en 3 subfases.

Terada y Matsumoto separaron el Cajamarca Cursivo Rectilíneo del Cajamarca Cursivo Clásico, pero el criterio que clasifica a los 2 no es claro y existen varios motivos combinados, así que lo incluimos en el Cajamarca Cursivo Clásico. La nueva cronología de la fase Cajamarca Media se resume de la siguiente manera: en la fase Cajamarca Media A se produjeron el Cajamarca Cursivo Clásico, en la fase Cajamarca Media B apareció el Cajamarca Cursivo Floral y en la fase Cajamarca Media C el Cajamarca Cursivo Floral se volvió más tosco y aparecieron por primera vez trípodes cortos en la cerámica caolín. El Complejo Turístico Baños del Inca se construyó en gran escala en la fase Cajamarca Media A, antes que apareciera el Cajamarca Cursivo Floral.

Daniel Julien, adoptando la cronología de Terada \& Matsumoto, dice lo siguiente: «La abundancia del Cajamarca Cursivo Floral en relación al Cajamarca Cursivo Clásico varía marcadamente de sitio a sitio. En algunos sitios, el Cursivo Floral está casi totalmente ausente. Uno de estos sitios es Cerro Coyor» (Julien, 1988: 161; la traducción es mía).

Según la nueva cronología se puede decir que la ausencia del Cajamarca Cursivo Floral no presenta variedad local, sino temporal y que Coyor pertenece a la fase Cajamarca Media A al igual que el Complejo Turístico Baños del Inca.

El tipo Cajamarca Cursivo Clásico se distribuye en un área amplia hasta Huamachuco (McCown, 1945; Thatcher, 1972; 1975) pero el Cajamarca Cursivo Floral se distribuye en un área más amplia aún como lo discutiremos más adelante.

\section{2. La fase Cajamarca Temprana}

La cerámica caolín de esta fase se caracteriza por el engobe de color naranja y la encontramos en un área amplia. Se puede decir que la variedad de la cerámica caolín es una característica de esta fase; aparte de «Cajamarca Pintado Lineal», existían 4 grupos representativos de su tradición alfarera: «Complejo Kolguitín», «Complejo Huacariz», «Wayrapongo Policromo» y «Cajamarca Precursivo». Terada \& Matsumoto subdividen la fase Cajamarca Temprana en 3 subfases, sugiriendo que en la subfase Cajamarca Temprana A surgieron el «Cajamarca Pintado Lineal» y el «Complejo Huacariz», en la subfase Cajamarca Temprana B el «Wayrapongo Policromo», y en la subfase Cajamarca Temprana C el «Complejo Kolguitín» y el «Cajamarca Precursivo». En la subfase C, es decir, al final de la fase Cajamarca Temprana, coexisten todos los tipos de cerámica caolín de la fase Cajamarca Temprana (Terada \& Matsumoto, 1985: 77). Esta interpretación queda por definir. 
Terada \& Matsumoto dicen que:

«Cabe señalar que la diferencia entre Colquitín ${ }^{6}$ y Amoshulca, en cuanto se refiere a la frecuencia de cerámica del tipo Cajamarca Precursivo y del Complejo Colquitín, en la misma subfase C, parece indicar que existían diferencias locales en un área relativamente pequeña» (Terada \& Matsumoto, 1985: 77).

Ellos indican la posibilidad de que la variabilidad del porcentaje de los 2 tipos (el Cajamarca Precursivo y el Complejo Kolguitín) represente la diferencia local. Es evidente la continuidad del Cajamarca Precursivo en el Cajamarca Cursivo Clásico tanto estratigráfica como estilísticamente, así que el Cajamarca Precursivo parece haber seguido produciéndose durante más tiempo que el Complejo Kolguitín, aunque los 2 empezaran a producirse al mismo tiempo. Julien también manifiesta que:

«(el Cajamarca Precursivo) es claramente transicional estilísticamente entre el Kolguitín Polícromo (un tipo del Complejo Kolguitín) y el Cajamarca Cursivo Clásico (...) el Cajamarca Precursivo es intermedio entre los dos tipos en virtualmente todos los atributos, desde forma de vasija, engobe, motivos decorativos, hasta su manera de aplicación» (Julien, 1988: 76; la traducción es mía).

Queda por definir en detalle la cronología de la fase Cajamarca Temprana. Quisiera recalcar que los 2 tipos recuperados en el mismo estrato no necesariamente comenzaron y terminaron de producirse al mismo tiempo.

\section{3. Descripción de la cerámica caolín}

De manera resumida, presentamos a continuación las características de los tres tipos de cerámica caolín.

\section{3. 1. Cajamarca Precursivo (fig. 5)}

Pasta: Es compacta, de color naranja y no se nota temperante. Se aplica el engobe del color naranja en el caso de la pasta de color blanco.

Acabado: La superficie está pulida horizontalmente.

Color: el engobe tiene el color naranja y se decora con negro y rojo.

Forma: Predomina la taza de base anular con la pared inclinada un poco hacia afuera. La pared tiene 3,5-4,5 cm de altura. Se notan las paredes en forma escalonada con uno o dos peldaños (fig. 5: A-I) y a veces en forma de zigzag (fig. 5: J, K).

Decoración: Hay variedad de decoración y se notan 3 grupos principales, entre los que destacan:

\footnotetext{
${ }^{6}$ Luego se adopta la ortografía de Kolguitín (Matsumoto, 1993).
} 
- El lado exterior se divide en 3 ó 4 bandas horizontales, cada una de los cuales se dibuja con línea corta y punto triangular (fig. 5: A- F). Se representa un motivo como un animal en el lado interior (fig. 5: F).

- En el lado exterior se representan unos círculos concéntricos, de los cuales salen unas líneas en forma radial (fig. 5: G, H, I). Además aparecen líneas diagonales, puntos triangulares y puntos pequeños. En el lado interior se representan líneas rojas y negras con puntos negros en ambos lados de la línea. Este grupo de decoración se limita a la forma con la pared escalonada.

- En el lado exterior dibujos de punto y círculo como un ojo, los mismos que aparecen solamente en la pared escalonada en forma de zigzag (fig. 5: J, K).

\section{3. 2. Cajamarca Cursivo Clásico (figs. 6-7)}

Pasta: Es muy compacta y no se nota temperante. Tiene el color blanco distinto al del Cajamarca Precursivo.

Acabado: Está muy bien pulida.

Color: La mayoría tiene el color blanco o crema, y a veces el color naranja. Se dibujan con 2 tipos de color: negro (o marrón) y rojo.

Forma: La única forma es la taza con base anular pequeña. Tiene la pared casi recta, inclinada un poco hacia fuera, y en muchos casos tiene la pared escalonada.

Decoración: Con líneas muy finas se dibujan la cara, el cuerpo de los hombres y los felinos en ambos lados. Se divide con líneas verticales, horizontales y diagonales. Todos tienen dibujos en la base del lado interior, mientras que algunos ejemplares no tienen dibujos en el lado interior de la pared.

Dentro del Cajamarca Cursivo Clásico hay un grupo que tiene el «motivo de radio7»), una decoración típica del Cajamarca Cursivo Floral8 (fig. 7), pero todavía no lo hemos clasificado como un tipo distinto. Se notan diferencias entre este grupo y el Cajamarca Cursivo Floral de la fase Cajamarca Media B (fig. 10) como se describe a continuación:

- Tiene la forma de la taza con la pared casi recta igual a la del Cajamarca Cursivo Clásico, mientras que el Cajamarca Cursivo Floral tiene la pared más gruesa e inclinada hacia afuera.

- Existen dobles líneas arriba y abajo del motivo de radio, mientras que el Cajamarca Cursivo Floral no tiene estas líneas.

- El ancho del motivo de radio es más estrecho que el del Cajamarca Cursivo Floral.

7 Spoke motif (o spoke design, spoke pattern) en inglés, usado por Thatcher (1972: 55; 1975: 118). este motivo se parece al radio de rueda.

8 En el informe de las excavaciones de Huacaloma, se incluyen los fragmentos de este grupo en Cajamarca Cursivo Clásico (Terada \& Onuki, 1982: PLATE 46a, 2-6, PLATE 104, 12-16, 22), y un fragmento en Cajamarca Cursivo Floral (Terada \& Onuki, 1982: PLATE 46b-11, PLATE 104-16). No se sabe en qué tipo se clasifica este grupo en el artículo de Terada \& Matsumoto (1985). Un fragmento se incluye dentro de Cajamarca Cursivo Rectilíneo (Matsumoto, 1993: fig. 13-2, b), que es idéntico a un fragmento de la LÁMINA 5 de Terada \& Matsumoto (1985). 
- Algunos ejemplares no tienen dibujos en el lado interior de la pared, mientras que el Cajamarca Cursivo Floral tiene dibujos en ambos lados.

En la fase Cajamarca Media A también existen algunos ejemplares que tienen las líneas paralelas horizontales en el lado exterior, con la misma forma que el Cajamarca Cursivo Clásico (Terada \& Onuki, 1982: PLATE 46a-11, PLATE 46b-12, PLATE 104-9, PLATE 104-11). Terada \& Matsumoto (1985: 78) lo incluyen dentro del Cajamarca Cursivo Floral, pero es de un tipo distinto que aparece en la fase Cajamarca Media A junto a Cajamarca Cursivo Clásico.

\section{3. 2. Cajamarca Cursivo Floral (fig. 10)}

Pasta: es fina pero más tosca que la de Cajamarca Cursivo Clásico; volviéndose más tosca con el tiempo.

Acabado: se aplica el engobe crema o blanco y se pule horizontalmente.

Color: tiene el color crema o blanco y se dibuja con pintura de color negro y rojo.

Forma: es la taza semiesférica o la taza con la pared inclinada hacia afuera, con base anular. En el periodo posterior (Cajamarca Media C) aparecen trípodes cortos.

Decoración: en el lado exterior se figura el «motivo de radio» y en el interior, aparecen los diseños geométricos o naturales como animales felinos con líneas curvas.

\section{EL CARÁCTER DE LA CULTURA CAJAMARCA Y EL ABANDONO DEL COMPLEJO TURÍSTICO BAÑOS DEL INCA}

La parte principal de la arquitectura del Complejo Turístico Baños del Inca se construyó en la fase Cajamarca Media A, cuando comenzaron a existir grandes centros claramente planificados, situación desapercibida en otras fases en el valle de Cajamarca. Un sitio representativo es Coyor, ubicado en un cerro a orillas del lago San Nicolás (figs. 1-2). Actualmente se encuentra muy destruido, pero se puede observar edificios de piedras trabajadas en la cima del cerro. En el dibujo de Charles Wiener, Coyor se presenta como un cerro amurallado con dos edificios rectangulares y uno oval en la cumbre (Wiener, 1993 [1880]: 139-141). Por su diseño parece haber sido un sitio ceremonial, igual que el Complejo Turístico Baños del Inca.

Es de notar que en otras fases no está marcada la presencia de centros planificados de gran escala. En la fase Cajamarca Media A se observa la alta calidad de la cerámica caolín y la complejidad de su decoración. Existe un paralelismo entre la escala de centros ceremoniales y la uniformidad y calidad de cerámica caolín, reforzando la idea de que la cerámica caolín tendría un carácter ceremonial. La fase Cajamarca Media A es la época en la cual aparecieron grandes sitios de carácter ceremonial, presentando un vínculo ritual fuerte para esta zona9.

9 Esta situación es similar a la del Nazca Temprano, que se integró alrededor del centro ceremonial Cahuachi y presenta cerámica uniforme (Silverman, 1993; 1994). 
Antes de la fase Cajamarca Media B el sitio Complejo Turístico Baños del Inca fue incendiado y destruido intencionalmente, y Coyor parece haber sido abandonado al mismo tiempo. Estos fenómenos habrían sido el resultado del cambio social causado por la combinación de diversos factores. Voy a mencionar aquí 2 de ellos relacionados con la prosperidad y decadencia de la cultura Cajamarca.

El primero es el cambio climático. El sitio se encontró al mismo nivel que las aguas subterráneas, presentando la posibilidad de que haya sido utilizado en una época más seca que la actual. También llama la atención el hecho de que el Complejo Turístico Baños del Inca esté ubicado cerca del río Chonta y Coyor queda a orillas del lago San Nicolás, mostrando así la importancia del acceso al agua. Según el resultado de las investigaciones de Quelccaya, sierra sur del Perú, la época era muy seca en los años 562-594 d.C. (Shimada et al., 1991). A pesar de no contar con un fechado C14 para el comienzo de la fase Cajamarca Media A, se supone que esta fase comienza en una época seca, alrededor de 550/600 d.C., mientras que Matsumoto da la fecha 450 d.C. porque él piensa que Cajamarca Media A es coetánea con Moche IV (Matsumoto, 1993: 174, 188). En caso de que sea válida nuestra interpretación, se plantea que cuanto menos seco se hace el clima, tanto más difícil se hace utilizar el terreno, porque sube el nivel de las aguas subterráneas. Cabe mencionar que en las excavaciones hechas en el Complejo Turístico Baños del Inca se encontró el piso de la plaza a una profundidad inferior al nivel de agua subterránea en época de lluvia.

Otro factor importante en lo que respecta al abandono del sitio está relacionado con la cultura Wari. Se han recuperado varios fragmentos del Cajamarca Cursivo Floral de la fase Cajamarca Media B en algunos sitios de la cultura Wari (Watanabe, 2002); pensamos que la transición de la fase Cajamarca Media A a la B tendría una relación con las actividades de los waris en el valle de Cajamarca. Parece que una ruta importante que tomaron los waris para bajar a la costa norte fue a través del valle de Cajamarca y del río Jequetepeque10, por lo tanto tenemos que establecer la correlación con la fecha exacta del comienzo de la presencia Wari a gran escala en la costa norte. De todas maneras, sería necesario situar el abandono del sitio a finales de la fase Cajamarca Media A en el contexto social de la época Wari.

\section{LA RELACIÓN CON WARI Y LA COSTA NORTE}

Izumi Shimada \& Matsumoto piensan que la fase Cajamarca Media A es contemporánea con Moche IV, la fase Cajamarca Media B con Moche V y Sicán Temprano (Lambayeque Temprano) y la fase Cajamarca Tardía con Sicán Medio11 (Shimada, 1987: 140; Matsumoto, 1993; Montenegro, 1997: 27).

10 Izumi Shimada menciona las supuestas rutas de Wari: Huarmey, Jequetepeque y probablemente, Santa (Shimada, 1994: 251).

11 Luego, Shimada describe la división entre la fase Cajamarca Media A y B en el 700 d.C., sugiriendo que la fase Cajamarca Media A y Moche V son contemporáneas (Shimada, 1999: 410-411). Además presenta un fragmento del Cajamarca Cursivo Clásico recuperado en Pampa Grande, a pesar de clasificarlo como Cajamarca Cursivo Floral (Shimada, 1994: 253, Figura 10.3). 
Sin embargo, no tenemos los datos directos que indiquen la contemporaneidad de la fase Cajamarca Media A y Moche IV. El autor piensa que la fase Cajamarca Media A en el valle de Cajamarca habría comenzado alrededor de 550/600 d.C. paralelamente al cambio climático.

El problema es el fechado de la transición de la fase Cajamarca Media A a la B y la fecha de la llegada de los waris al valle de Cajamarca. Se piensa que la fase Cajamarca Media A terminó alrededor o un poco después de 700 d.C. Tenemos que confirmar el fechado del periodo en el que apareció la cerámica de estilo Cajamarca como el Cajamarca Cursivo Floral en otra área. En el sitio arqueológico San José de Moro en el valle bajo del Jequetepeque, la primera aparición de la cerámica de estilo Wari coincide con los primeros ejemplares del estilo Cajamarca (Castillo, 2001: 325; 2002: 174) en el periodo Mochica Tardío antes del periodo Transicional (800-950 d.C.12). Así que la fecha de la llegada de los waris al valle de Cajamarca no debe ser más tardía que 800 d.C.; luego los waris bajaron a la costa norte a lo largo del río Jequetepeque. Se sabe que los waris llegaron al valle de Cajamarca a finales de la fase Cajamarca Media A, o a comienzos de la fase Cajamarca Media B, en base a datos indirectos como la cerámica caolín encontrada en los sitios Wari, pero todavía no tenemos datos directos que lo indiquen, como el fechado radiocarbónico por ejemplo.

En resumen, se piensa que alrededor de 550/600 d.C. ocurrieron fenómenos paralelos en los Andes Centrales: el desarrollo de las culturas Wari y Cajamarca en la fase Cajamarca Media A; y que alrededor del 700 d.C. o un poco antes, los waris llegaron a Cajamarca, en donde se pasó de la fase Cajamarca Media A a la $\mathrm{B}$, luego bajaron a la costa norte a lo largo del río Jequetepeque y allí entraron en contacto con la gente de la sociedad Mochica Tardío (Moche V). La relación cronológica se resume como se observa en el cuadro 2 a pesar de que los fechados varían entre las áreas. Las fases Cajamarca Media B y C (700-900 d.C.) serían aproximadamente contemporáneas con la última parte del periodo Mochica Tardío y el periodo Transicional (800-950 d.C.) en el valle bajo de Jequetepeque y Sicán Temprano (700-900 d.C.) en la zona Lambayeque (Shimada, 1995).

En el área Huamachuco al sur del valle de Cajamarca existe un centro administrativo Wari de gran escala que se llama Viracochapampa (Topic, 1991; Topic \& Lange Topic, 1983; 1985; Lange Topic, 1991; Lange Topic \& Topic, 1984). John R. Topic y Theresa Lange Topic insisten reiteradamente en que Viracochapampa dejó de construirse antes de que se terminara, sugiriendo que dicho sitio no fue utilizado intensamente. Estamos de acuerdo en que la construcción de Viracochapampa no fue terminada, pero tenemos que considerar el por qué.

Una posibilidad es, como piensan los Topic, que la relación entre Huamachuco y Wari acabó debilitando el dominio Wari en la sierra norte. La otra posibilidad es que Huamachuco dejó de ser un lugar estratégico en el dominio Wari, pero la sierra norte del Perú continuó bajo su dominio. El autor tiene una hipótesis según

12 Luis Jaime Castillo otorga al periodo Transicional del valle Jequetepeque la fecha 800-950 d.C. (Castillo, 2001: 327; 2002: 162), aunque no ha presentado fechado de C14. 
Cuadro 2 - Correlación cronológica

\begin{tabular}{|c|c|c|c|}
\hline Fecha (d.C.) & Cajamarca & $\begin{array}{l}\text { Jequetepeque } \\
\text { (Castillo, 2001) }\end{array}$ & $\begin{array}{c}\text { Lambayeque } \\
\text { (Shimada, 1995) }\end{array}$ \\
\hline $550 / 600-700$ & Cajamarca Media A & \multirow{2}{*}{ Mochica Tardío } & Moche V \\
\hline \multirow{2}{*}{$700-900$} & \multirow{2}{*}{ Cajamarca Media B y C } & & Sicán Temprano \\
\hline & & Transicional & \\
\hline $900-1200$ & Cajamarca Tardía & Lambayeque & Sicán Medio \\
\hline
\end{tabular}

la cual los waris concentraron su mano de obra en la zona Cajamarca después de abandonar Viracochapampa. En este caso tenemos que estudiar la relación con la cultura Moche. En estos años ha avanzado el estudio sobre la cultura Moche, y se sabe que no se puede aplicar la misma cronología para explicar la dinámica cultural en toda el área de Moche. En la región situada al sur de la Pampa de Paiján (Moche del Sur) la fase Moche IV continúa hasta 700 d.C. (Chapdelaine, 2001; 2002), mientras que en la zona de Moche del Norte la transición de la fase Moche IV a la fase Moche V correspondería a 550/600 d.C. y la Moche V termina alrededor de 700 d.C. (Shimada, 1994). Sería bueno separar los casos del norte y del sur para considerar la relación con la cultura Moche pero podría significar que Moche del Sur mantuvo su cultura más tiempo.

Lo importante es la ruta entre la sierra norte y la costa norte. Huamachuco es un lugar estratégico para bajar a la costa norte a través del río Moche o Chicama, y Cajamarca es el punto clave para llegar a la costa norte por el río Jequetepeque. Planteamos que los waris cambiaron su estrategia; primero conquistar o negociar con Moche del Norte antes que con Moche del Sur, haciendo más importante Cajamarca que Huamachuco. Según los Topic, Viracochapampa estaba en construcción en el Horizonte Medio 1B (MH1B) durante unos 50 años (Topic, 1991: 163; Lange Topic \& Topic, 1984: 56) entre 650-700 d.C. (Topic \& Lange Topic, 2001: 206). Además, como se discute abajo, se ha documentado cerámica del estilo Cajamarca en los sitios Wari en las fases Cajamarca Media B y C que sería posterior al abandono de Viracochapampa alrededor del 700 d.C.

De todas maneras mientras Viracochapampa estaba construyéndose, Huamachuco habría estado bajo el dominio Wari o tenía una relación íntima con los waris. Además se ha reportado una tumba u ofrenda en Cerro Amaru en el Horizonte Medio (Lange Topic \& Topic, 1984). En un mausoleo de $7 \times 7 \mathrm{~m}$ se ubicaron 
3 cámaras subterráneas en las que se registró una tumba u ofrenda. Se encontraron una cerámica del Cajamarca Cursivo Floral con incisión en forma de X en la base del lado exterior, y 3 cucharas de la cultura Cajamarca junto con cerámica de múltiples orígenes como por ejemplo de la cultura Wari.

Los Topic piensan que se reutilizó el edificio que ya había existido en Cerro Amaru. Los fechados de carbono 14 indican la fecha del Periodo Intermedio Temprano, unos siglos antes de la llegada de los waris. Lamentablemente el único fechado UGa 4865 obtenido de una cámara subterránea presenta un fechado muy antiguo para ser de la época Wari, y según la interpretación de los Topic habría sido causado por la introducción de materiales de los niveles superiores a los inferiores (Lange Topic \& Topic, 1984: 74-76). Estamos de acuerdo con los Topic en que la arquitectura de Cerro Amaru se reutilizó en el Horizonte Medio a modo de ofrenda. Los Topic piensan que el mausoleo de Cerro Amaru se incendió a comienzos de Horizonte Medio 2A (Topic \& Lange Topic, 1992: 172), que es casi contemporáneo con el abandono del Complejo Turístico Baños del Inca; también es importante Cerro Amaru porque sería una muestra de cambio climático (Topic \& Lange Topic, 1992: 174), igual que el caso del Complejo Turístico Baños del Inca.

Se han documentado ofrendas semejantes, por ejemplo, en el Sitio A del complejo de Huaca del Sol de Moche donde se encontró cerámica Cajamarca Cursivo Floral con trípode de la fase Cajamarca Media C (Kroeber, 1925: 212-213), posterior a la primera evidencia de la presencia Wari en el valle bajo de Jequetepeque. La ofrenda con objetos de múltiples orígenes en el Horizonte Medio en muchos casos se realizó en el sitio abandonado, como una dedicatoria a las huacas.

En la nueva cronología de la fase Cajamarca Media presentada en este artículo, la fase Cajamarca Media B y C (700-900 d.C.) corresponde a la época Wari. Lo interesante de las fases Cajamarca Media B y C reside en que el tipo Cajamarca Cursivo Floral se distribuye en un área amplia tanto en la sierra como en la costa de la zona de Moche del Norte. En algunos sitios Wari como Jargampata, Conchopata y Huari mismo, se han encontrado algunos fragmentos de cerámica de la cultura Cajamarca, la mayoría de los cuales son Cajamarca Cursivo Floral (Watanabe, 2002). Hay variedad de la cerámica de estilo Cajamarca en el valle bajo de Jequetepeque como el Cajamarca Cursivo Floral con base anular y la cerámica con trípode corto (de la fase Cajamarca Media C); por lo tanto la cerámica Cajamarca aparece en el valle bajo de Jequetepeque durante un periodo mayor al de los sitios de la cultura Wari de la sierra donde no se ha encontrado la cerámica caolín con trípode.

Se ha explicado que la amplia distribución de la cerámica Cajamarca Cursivo Floral se debe a la prosperidad de la cultura Cajamarca o a su relación con la cultura Wari; por ejemplo, Luis G. Lumbreras dice sobre Wari que:

«En la sierra, en el extremo septentrional, la conquista incluyó la región de Cajamarca, que seguramente jugó un rol de importancia dentro de la cultura Wari, pues, por ejemplo, su cerámica tuvo mucho prestigio, fue utilizada en el sitio mismo de Wari y se distribuyó a varios lugares, entre ellos Moche» (1969: 260; 1974: 168). 
Los Topic piensan que la cerámica caolín de la cultura Cajamarca sería un indicador de prestigio de dicha cultura (Topic \& Lange Topic, 2001: 186-187). Por otro lado, Terada \& Matsumoto refieren que:

«sería peligroso atribuir a priori el cambio cultural de Cajamarca a la expansión y decadencia de la cultura Huari como se supone en general» (Terada \& Matsumoto, 1985: 88).

Para entender el mecanismo de la distribución de la cerámica Cajamarca, tenemos primero que considerar si es normal que se intercambiara la cerámica por sí, como fue el caso del Spondylus u obsidiana, o que los ceramistas mismos se movilizaran13.

Creo que la distribución de la cerámica Cajamarca indicaría un fenómeno semejante al de la cerámica Chimú y Chimú-Inca en la época del Tawantinsuyu. Menzel ha mencionado la posibilidad de que hubiese una colonia de cajamarquinos en el sitio Huari tal como la unidad militar de los cañares y chachapoyas en el Cuzco durante el Tawantinsuyu (Menzel, 1964: 72). El autor piensa que la amplia distribución de la cerámica Cajamarca Cursivo Floral no se debe al intercambio de cerámica (Lau, 2006), sino al movimiento de la gente cajamarquina como si fuesen «mitimaes» bajo el dominio del estado Wari (Watanabe, 2002: 538). Mi interpretación se refuerza por la presencia del sitio El Palacio en el valle de Cajamarca, pues se trataría de un centro administrativo Wari (Lumbreras, 1969: 261; 1974: 171; Watanabe, 2002); además 3 cucharas de caolín encontradas en Jargampata (Isbell, 1977: 118) indicarían que se introdujo una costumbre de la cultura Cajamarca, que consistía en usar la cerámica caolín junto con cucharas. Para comprobar mi hipótesis, tenemos primero que averiguar si la cerámica del estilo Cajamarca Cursivo Floral ha sido hecha en el valle de Cajamarca y exportada a otra zona, o hecha en otras áreas, y luego buscar otras características que indiquen la presencia de los cajamarquinos: la cerámica no caolín tal como el Cajamarca Rojo Tosco, la arquitectura y tumbas si suponemos que hubo inmigrantes permanentes ${ }^{14}$.

En el caso en que la cerámica caolín por sí se haya importado, su lugar de producción habría estado fuera del valle de Cajamarca, porque sabemos que la mayoría de la cerámica caolín recuperada presenta características distintas a la del valle de Cajamarca, aunque no se puede rechazar la posibilidad que algunos ejemplares del estilo Cajamarca se hayan importado directamente del valle de Cajamarca.

13 La presencia de Spondylus no indica la presencia de los ecuatorianos; pues Spondylus u obsidiana no se movilizan como objeto cultural, sino como materia prima. La cultura Cajamarca se define por la producción de la cerámica caolín, pero hasta hoy no se ha documentado la existencia de una cultura que se defina por el empleo de Spondylus u obsidiana en el Ecuador.

14 También se ha encontrado cerámica del estilo Cajamarca en Marca Huamachuco (McCown, 1945), del Cajamacra Cursivo Clásico de la fase Cajamarca Media A, contemporánea con la construcción Viracochapampa, donde se han encontrado asas con diseños incisos del Cajamarca Rojo Tosco de la cultura Cajamarca (Topic \& Lange Topic, 1983: 23), al igual que en Cerro Amaru (Lange Topic \& Topic, 1984: 46; Topic \& Lange Topic, 1985: 32). Estos datos significarían que los cajamarquinos iban a Huamachuco, bajo el dominio Wari, cuando la zona Cajamarca ya se encontraba bajo el dominio Wari en la fase Cajamarca Media A, alrededor de 650 d. C., a la par con el caso de Huamachuco. 
La otra posibilidad es la influencia estilística: la gente exterior al valle de Cajamarca imitó la cerámica caolín de la cultura Cajamarca. Sin embargo, hasta ahora no se ha encontrado cerámica Cajamarca con características de fusión con la cerámica de otra cultura; si la gente de fuera del valle de Cajamarca hubiese imitado la cerámica caolín, podría haber más diversidad entre los sitios. Así que el autor piensa que lo fabricó la gente del mismo lugar de origen y es más probable que los cajamarquinos mantuviesen la misma tradición alfarera fuera del valle de Cajamarca.

Por último, para entender la dinámica cultural ocurrida en la fase Cajamarca Media quisiéramos hacer referencia a un grupo de cerámica denominado en general «Cajamarca Costeño», porque presenta similitud con la del valle de Cajamarca. Cajamarca Costeño no presenta similitud con el Cajamarca Cursivo Floral, sino más bien con la cerámica no caolín de dibujo geométrico y de color rojo de la sierra15.

Se trata de platos o tazas con base anular o trípode, con dibujos geométricos de color rojo sobre la pintura blanca en el lado interior y pintados como una banda en color blanco cerca del borde en el lado exterior; no son importados, sino elaborados en el lugar con la arcilla no caolín de color anaranjado. Hasta ahora se han encontrado en la costa norte, sobre todo en el Jequetepeque hacia el lado norte, o sea en la zona del Moche del Norte, y en el valle medio de Jequetepeque. Es de notar que la cerámica Cajamarca Costeño no solamente aparece en los contextos funerarios, sino también en los contextos domésticos como es el caso de la cerámica caolín en el valle de Cajamarca.

Jorge Montenegro realizó el estudio de este grupo de cerámica, encontrando que el grupo Cajamarca Costeño aparece inmediatamente encima de la capa de Moche $V$ en el valle medio de La Leche, y al final del Moche $V$ o justo después de la desaparición de la cerámica de estilo Moche en el valle bajo de Jequetepeque; prosigue en Sicán Medio en la zona Lambayeque o el periodo Lambayeque en el valle bajo de Jequetepeque, pero no aparece en Sicán Tardío (1100-1375 d.C.) (Montenegro, 1997: 43-49). Revisando los datos del valle de Cajamarca, se puede decir que el Cajamarca Costeño apareció al comienzo de la fase Cajamarca Media B y siguió produciéndose en las fases Cajamarca Media C y Cajamarca Tardía y desapareció en la costa norte al final de la fase Cajamarca Tardía.

Shimada menciona que se habría establecido una colonia Cajamarca en Lambayeque en la fase Cajamarca Media (Shimada, 1994: 251, 259). El término «colonia» ha sido utilizado por John V. Murra para plantear el modelo de «control vertical» (Murra, 1972). Y si existía la colonia cajamarquina en Lambayeque, podría decir que esta fue posible bajo el dominio de una organización política de gran

15 Este grupo de cerámica no se considera como un «tipo» determinado. Bernuy \& Bernal (2005) se refieren a este grupo como parte del Cajamarca Cursivo Clásico, tomando un ejemplo de Huacaloma (Terada \& Onuki, 1982: PLATE 105-6); pero no es tan cierta dicha clasificación. Esta cerámica se distribuye dentro del valle de Cajamarca como fuera de él. Según las excavaciones realizadas en la temporada 2006 en el sitio arqueológico Paredones, ubicado en el valle medio de Jequetepeque, esta cerámica se fabrica en la fase Cajamarca Media B, junto con el Cajamarca Cursivo Floral, y se ha denominado Cajamarca Rojo Pintado (Watanabe, 2007). 
escala como Wari o más probable con Sicán Medio por la proximidad geográfica y el intercambio fuerte de cobre y otros metales. Esta interpretación concuerda con el caso de la sierra sur, el cual indica que la colonia de los grupos étnicos del altiplano en los valles bajos, como Lupaca en Moquegua, no fue el resultado del movimiento del grupo independiente, sino que fue posible bajo el dominio del Tawantinsuyu (Van Buren, 1996; Stanish, 2003) o Tiwanaku (Goldstein, 2005).

Cajamarca Costeño, a pesar de que existe similitud con la cerámica Cajamarca, es un grupo muy distinto al de la cerámica de la sierra. Su origen no se encuentra en el valle de Cajamarca, sino en la costa norte o en el valle medio o bajo de Jequetepeque. El grupo Cajamarca Costeño no desapareció al mismo tiempo que Wari en la costa norte, sino que continuó produciéndose en la época siguiente, Sicán Medio, que correspondía a la fase Cajamarca Tardía en el valle de Cajamarca. El caso de la zona de Lambayeque significaría que los que producían Cajamarca Costeño trasladados en la época Wari (Sicán Temprano) se incorporaron o se quedaron bajo el dominio Sicán Medio después del retiro de la presencia Wari en la región de Cajamarca. Parece que los descendientes de la gente que empezaron a usar la cerámica Cajamarca Costeño permanecieron en la costa norte, manteniendo su propio estilo de cerámica que representaría su identidad cultural. Creo que Cajamarca Costeño no es solamente un estilo de cerámica, sino que indicaría la existencia de un grupo humano. Si es válida esta interpretación, se supone que los que fabricaron la cerámica Cajamarca Costeño perdieron su identidad cultural o se mudaron a otro lugar cuando Cajamarca Costeño desapareció durante el Sicán Tardío. Se parece al caso de Gallinazo que subsistió en los ríos Jequetepeque y La Leche en el periodo Moche (Shimada, 1994: 66-71). También es muy sugestivo mencionar que en la parte sur del Perú en la época colonial los descendientes de mitimaes de la época incaica se quedaron en el enclave (colonia) para alcanzar su objetivo político (Van Buren, 1996). Sería muy ilustrativo como modelo el caso de los Chachapoyas y Cañaris, mitimaes bajo el dominio Tawantinsuyu, quienes mantuvieron la identidad étnica en el Cuzco en la época colonial (Dean, 1999).

Falta aclarar si Cajamarca Costeño durante la fase Sicán Medio fue un fenómeno independiente de la sierra. Se sabe que en el valle de Cajamarca disminuye el número de los sitios en la fase Cajamarca Tardía (Terada \& Matsumoto, 1985; Julien, 1988). Creo que podría haber una correlación entre la situación del valle de Cajamarca y la prosperidad del Sicán Medio. Terada y Matsumoto refieren que es dominante el tipo Cajamarca Negro y Anaranjado de la fase Cajamarca Tardía en Chota y Tacabamba al norte del valle de Cajamarca (Shady \& Rosas, 1977; Terada \& Matsumoto, 1985: 83). Es posible que hubiera un movimiento de gran cantidad de gente hacia la zona más al norte del valle de Cajamarca, el lugar próximo a Batán Grande que es el centro de Sicán Medio. En ese caso, tenemos que tener en cuenta la posibilidad de la explotación de los materiales como minerales, sobre todo el cobre $(\mathrm{Cu})$ y el oro $(\mathrm{Au})$. Pero aún carecemos de datos de la zona norte fuera del valle de Cajamarca. 


\section{COMENTARIOS FINALES}

En este artículo hemos propuesto una nueva cronología de la fase Cajamarca Media en base a los datos de excavaciones en el Complejo Turístico Baños del Inca y consideramos la relación de la cultura Cajamarca con otras culturas. La cultura Cajamarca produjo sin cesar cerámica caolín durante 1600 años, pero no era una cultura estática, al tener una historia muy dinámica manteniendo relaciones con otras sociedades.

Cada cultura tiene su propio valor y a él se dedican las labores humanas. En muchas culturas andinas prehispánicas se estimó a la cerámica, y por eso la arqueología establece una unidad cultural en base a la cerámica. Por ello, la cultura que tiene una cerámica diagnóstica se hace famosa, a pesar de que existan culturas que se dediquen a otras actividades como la arquitectura. La cerámica caolín caracteriza a la cultura Cajamarca, y a los cajamarquinos. La fase Cajamarca Media A presenta en el Cajamarca Cursivo Clásico la máxima calidad tanto en la pasta como en la minuciosidad de los dibujos. Su desarrollo cultural autóctono cesó por el dominio de la sociedad Wari, pero mantuvo su propia tradición de cerámica caolín, a pesar de que hubo un cambio estilístico. Su situación presenta un contraste con la cultura Moche que terminó alrededor del 800 d.C. También tenemos que considerar la flexibilidad de la cultura Cajamarca teniendo en cuenta su estructura social.

Hasta ahora el estudio de la cultura Cajamarca está basado en la cerámica caolín, pero para ir esclareciendo las características de dicha cultura, es necesario tener datos sobre la arquitectura, el patrón funerario, etc. Además queda pendiente el estudio de la iconografía de la cerámica caolín, por lo tanto aún queda mucho por investigar.

\section{Agradecimientos}

Este trabajo se realizó gracias al apoyo del Pache Research Fund I-A-2 del 2009 de la Nanzan Universidad. Las excavaciones en el Complejo Turístico Baños del Inca se realizaron bajo los auspicios de la Comisión Administrativa del Complejo Turístico Baños del Inca. Mi profunda gratitud a los miembros del proyecto, Juan Ugaz, Evelyn Anyanett Mora Coronado, Regina Abraham, Jacqueline Tejada Bobadilla y Carlos del Mar. Quisiera agradecer a Juan Ugaz y Tomás Campos por revisar el texto, a Shigeru Takeuchi y Taiga Wakabayashi por preparar los dibujos, a John Boccellari y Patrick Rebollar por traducir los resúmenes, y también reconocer los comentarios y observaciones valiosos de Theresa Lange Topic y George Lau, al mismo tiempo la evaluación anónima del boletín Corriente Arqueológica. Este trabajo se basa en la ponencia que presenté en el IV Seminario de Arqueología UNFV: La producción y distribución de cerámica como indicador social y político en los Andes prehispánicos y coloniales; agradezco a los organizadores del simposio por invitarme al evento. 


\section{Referencias citadas}

BERNUY, K. \& BERNAL, V., 2005 - Influencia Cajamarca en los rituales funerarios del periodo Transicional en San José de Moro. Corriente Arqueológica, 1: 61-77.

BRONK RAMSEY, C., 1995 - Radiocarbon calibration and analysis of stratigraphy: The OxCal program. Radiocarbon, 37 (2): 425-430.

BRONK RAMSEY, C., 2001 - Development of the radiocarbon calibration program OxCal. Radiocarbon, 43 (2A): 355-363.

BRONK RAMSEY, C., 2006 - OxCal v. 4.0.1.

CASTILLO, L. J., 2001 - The Last of the Mochicas: A View from the Jequetepeque Valley. In: Moche Art and Archaeology in Ancient Peru (J. Pillsbury, ed.): 306-332; Washington: National Gallery of Art.

CASTILLO, L. J., 2002 - La presencia de Wari en San José de Moro. Boletín de Arqueología PUCP, 4: 143-179.

CHAPDELAINE, C., 2001 - The Growing Power of a Moche Urban Class. In: Moche Art and Archaeology in Ancient Peru (J. Pillsbury, ed.): 68-87; Washington: National Gallery of Art.

CHAPDELAINE, C., 2002 - Out in the Streets of Moche: Urbanism and Sociopolitical Organization at a Moche IV Urban Center. In: Andean Archaeology I: Variations in Sociopolitical Organization (W. H. Isbell \& H. Silverman, eds.): 53-88. New York: Kluwer Academic, Plenum Publishers.

DEAN, C., 1999 - Inka Bodies and the Body of Christ: Corpus Christi in Colonial Cuzco, Peru, 288 pp.; Durham: Duke University Press.

GOLDSTEIN, P. S., 2005 - Andean Diaspora: The Tiwanaku Colonies and the Origins of South American Empire, 403 pp.; Gainesville: University Press of Florida.

GRIEDER, T., 1978 - The Art and Archaeology of Pashash, 268 pp.; Austin: University of Texas Press.

ISBELL, W. H., 1977 - The Rural Foundations for Urbanism: Economic and Stylistic Interaction between Rural and Urban Communities in Eighth-Century Peru, 188 pp.; Urbana: University of Illinois Press.

KROEBER, A. L., 1925 - The Uhle Pottery Collections from Moche. University of California Publications in American Archaeology and Ethnology, 21 (5): 191-234.

JULIEN, D. G., 1988 - Ancient Cuismancu: Settlement and Cultural Dynamics in the Cajamarca Region of the North Highlands of Peru, 394 pp.; Austin: Department of Anthropology, University of Texas. Ph.D. dissertation.

LANGE TOPIC, T., 1991 - The Middle Horizon in Northern Peru. In: Huari Administrative Structure: Prehistoric Monumental Architecture and State Government (W. H. Isbell \& G. F. McEwan, eds.): 233-246; Washington, D.C.: Dumbarton Oaks Research Library and Collection.

LANGE TOPIC, T. \& TOPIC, J. R., 1984 - Huamachuco Archaeological Project: Preliminary Report of the Third Field Season, June-August 1983, 80 pp. con 13 figuras; Peterborough, Ontario: Department of Anthropology, Trent University. Trent University Ocasional Papers in Anthropology 1.

LANGE TOPIC, T. \& TOPIC, J. R., 1987 - Huamachuco Archaeological Project: Preliminary Report on the 1986 Field Season; Peterborough, Ontario: Department of Anthropology, Trent University.

LAU, G. F., 2006 - Northern Exposures: Recuay-Cajamarca Boundaries and Interaction. 
In: Andean Archaeology III: North and South (W. H. Isbell \& H. Silverman, eds.): 143-170; New York: Springer.

LUMBRERAS, L. G., 1969 - De los Pueblos, las Culturas y las Artes del Antiguo Perú, 377 pp.; Lima: Moncloa-Campodonico, Editores Asociados.

LUMBRERAS, L. G., 1974 - The Peoples and Cultures of Ancient Peru, 248 pp.; Washington: Smithsonian Institution Press. Translated by B.J. Meggers.

MATSUMOTO, R., 1993 - Dos modos de proceso socio-cultural: el Horizonte Temprano y el Periodo Intermedio Temprano en el valle de Cajamarca. In: El Mundo Ceremonial Andino (L. Millones \& Y. Onuki, eds.): 169-202; Senri Ethnological Studies n. ${ }^{\circ} 37$. Osaka: National Museum of Ethnology.

MCCORMAC, F. G., HOGG, A. G., BLACKWELL, P. G., BUCK, C. E., HIGHAM, T. F. G. \& REIMER, P. J., 2004 - SHCal04 Southern Hemisphere Calibration, 0-11.0 cal kyr BP. Radiocarbon, 46 (3): 1087-1092.

McCOWN, T. D., 1945 - Pre-Incaic Huamachuco: Survey and Excavations in the Region of Huamachuco and Cajabamba. University of California Publications in American Archaeology and Ethnography, 39 (4): 223-399.

MENZEL, D., 1964 - Style and Time in the Middle Horizon. Nawpa Pacha, 2: 1-105.

MONTENEGRO CABREJO, J. A., 1997 - Coastal Cajamarca Pottery from the North Coast of Peru: Style, Technology, and Function, 335 pp.; Department of Anthropology, Southern Illinois University. Tesis de Maestría.

MURRA, J. V., 1972 - El «Control vertical» de un máximo de pisos ecológicos en la economía de las sociedades andinas. In: Visita de la Provincia de León de Huánuco en 1562, por Iñigo Ortiz de Zúñiga (J. V. Murra, ed.): 429-476. Documentos para la Historia y Etnología de Huánuco y la Selva Central, vol. 2; Huánuco: Universidad Nacional Hermilio Valdizán.

ONUKI, Y. (ed.), 1995 - Kuntur Wasi y Cerro Blanco: Dos Sitios del Formativo en el Norte del Perú, 217 pp.; Tokio: Hokusen-sha.

OYUELA-CAYCEDO, A., 1995 - Rocks versus Clay: The Evolution of Pottery Technology in the Case of San Jacinto 1, Colombia. In: The Emergence of Pottery: Technology and Innovation in Ancient Societies (W. K. Barnett \& J. W. Hoopes, eds.): 133-144; Washington, London: Smithsonian Institution Press.

RAVINES, R., 1985 - Cajamarca Prehispánica: Inventario de Monumentos Arqueológicos, 142 pp.; Cajamarca: Instituto Nacional de Cultura de Cajamarca, Corporación de Desarrollo de Cajamarca. Inventarios del Patrimonio Monumental del Perú 2.

REICHLEN, H. \& REICHLEN, P., 1949 - Recherches archéologiques dans les Andes de Cajamarca : premier rapport de la Mission Ethnologique Française au Pérou Septentrional. Journal de la Société des Américanistes, 38: 137-174.

REYCRAFT, R. M. (ed.), 2005 - Us and Them: Archaeology and Ethnicity in the Andes, 242 pp.; Los Angeles: Cotsen Institute of Archaeology, University of California. Monograph 53.

ROOSEVELT, A. C., 1995 - Early Pottery in the Amazon: Twenty Years of Scholarly Obscurity. In: The Emergence of Pottery: Technology and Innovation in Ancient Societies (W. K. Barnett \& J. W. Hoopes, eds.): 115-131; Washington, London: Smithsonian Institution Press.

SCHJELLERUP, I. R., 1997 - Incas and Spaniards in the Conquest of the Chachapoyas: Archaeological and Ethnohistorical Research in the North-eastern Andes of Peru, 343 pp.; Göteborg: Department of Archaeology, Göteborg University. GOTARC Series B. Gothenburg Archaeological Theses No 7. 
SEKI, Y. \& TEJADA, C., 2003 - Informe Preliminar del Proyecto de Investigaciones Arqueológicas en el valle de Cajamarca, Peru, Temporada 2003; Lima: INC.

SEKI, Y. \& UGAZ, J., 2002 - Informe Preliminar del Proyecto de Investigaciones Arqueológicas en el valle de Cajamarca, Peru, Temporada 2002; Lima: INC.

SEKI, Y., UGAZ, J. \& WATANABE, S., 2001 - Informe Preliminar del Proyecto de Investigaciones Arqueológicas en el Valle de Cajamarca, Perú; Lima: INC.

SHADY, R. S., \& ROSAS, H., 1977 - El Horizonte Medio en Chota: prestigio de la cultura Cajamarca y su relación con el «lmperio Wari». Arqueológicas, 16: 1-75.

SHIMADA, I., 1987 - Horizontal and Vertical Dimensions of Prehistoric States in North Peru. In: The Origins and Development of the Andean State (J. Haas, S. Pozorski \& T. Pozorski, eds.): 130-144; Cambridge: Cambridge University Press.

SHIMADA, I., 1994 - Pampa Grande and the Mochica Culture, 323 pp.; Austin: University of Texas Press.

SHIMADA, I., 1995 - Cultura Sicán: Dios, Riqueza y Poder en la Costa Norte del Perú, 219 pp.; Lima: Fundación del Banco Continental para el Fomento de la Educación y la Cultura, Edubanco.

SHIMADA. I., 1999 - Evolution of Andean Diversity: Regional Formations (500 B.C.E.-C.E. 600). In: The Cambridge History of the Native Peoples of the Americas (F. Salomon \& S. B. Schwartz, eds.), vol. III: South America, Part 1: 350-517; Cambridge: Cambridge University Press.

SHIMADA, I., SCHAAF, C. B., THOMPSON, L. G. \& MOSLEY-THOMPSON, E., 1991 Cultural Impacts of Severe Droughts in the Prehistoric Andes: Application of a 1,500-year Ice Core Precipitation Record. World Archaeology, 22 (3): 247-270.

SILVERMAN, H., 1993 - Cahuachi in the Ancient Nasca World, 371 pp.; lowa City: University of lowa Press.

SILVERMAN, H., 1994 - The Archaeological Identification of an Ancient Peruvian Pilgrimage Center. World Archaeology, 26 (1): 1-18.

STANISH, C., 2003 - Ancient Titicaca: The Evolution of Complex Society in Southern Peru and Northern Bolivia, 354 pp.; Berkeley: University of California Press.

TERADA, K. \& MATSUMOTO, R., 1985 - Sobre la cronología de la tradición Cajamarca. In: Historia de Cajamarca 1: Arqueología (F. Silva Santisteban, W. Espinoza Soriano \& R. Ravines, eds.): 67-89; Cajamarca: Instituto Nacional de Cultura-Cajamarca.

TERADA, K. \& ONUKI, Y. (eds.), 1982 - Excavations at Huacaloma in the Cajamarca Valley, Peru, 1979, 351 pp. with 135 plates; Tokyo: University of Tokyo Press.

TERADA, K. \& ONUKI, Y. (eds.), 1985 - The Formative Period in the Cajamarca Basin, Peru: Excavations at Huacaloma and Layzon, 1982, 345 pp. with 146 plates; Tokyo: University of Tokyo Press.

THATCHER, J. P., 1972 - Continuity and Change in the Ceramics of Huamachuco, North Highlands, Peru, 258 pp.; Faculty of the Graduate School of Arts and Sciences, University of Pennsylvania. Ph.D. dissertation.

THATCHER, J. P., 1975 - Early Intermediate Period and Middle Horizon 1B ceramic assemblages of Huamachuco, north highlands, Peru. Nawpa Pacha, 10-12: 109-127.

TOPIC, J. R., 1991 - Huari and Huamachuco. In: Huari Administrative Structure: Prehistoric Monumental Architecture and State Government (W. H. Isbell \& G. F. McEwan, eds.): 141-164; Washington D.C.: Dumbarton Oaks Research Library and Collection.

TOPIC, J. R. \& LANGE TOPIC, T., 1983 - Huamachuco Archaeological Project: Preliminary Report of the Second Season, June-August 1982; Peterborough, Ontario: Department of Anthropology, Trent University. 
TOPIC, J. R. \& LANGE TOPIC, T., 1985 - El Horizonte Medio en Huamachuco. Revista del Museo Nacional, 47: 13-52.

TOPIC, J. R. \& LANGE TOPIC, T., 1992 - The Rise and Decline of Cerro Amaru: An Andean Shrine during the Early Intermediate Period and Middle Horizon. In: Ancient Images, Ancient Thought: The Archaeology of Ideology (A. S. Goldsmith, S. Garvie, D. Selin \& J. Smith, eds.): 167-180; Calgary: Archaeological Association, University of Calgary.

TOPIC, J. R. \& LANGE TOPIC, T., 2001 - Hacia la comprensión del fenómeno Huari: una perspectiva norteña. Boletín de Arqueología PUCP, 4 (2000): 181-217.

VAN BUREN, M., 1996 - Rethinking the Vertical Archipelago: Ethnicity, Exchange, and History in the South Central Andes. American Anthropologist, 98 (2): 338-351.

WATANABE, S., 2002 - Wari y Cajamarca. Boletín de Arqueología PUCP, 5: 531-541.

WATANABE, S., 2007 - Excavaciones en el sitio Paredones, sierra norte del Perú. América Antigua, 10: 67-98.

WATANABE, S., MORA CORONADO, E. A. \& MAR PACHECO, C. del, 2003 - Informe del Proyecto de Investigación Arqueológica y Conservación en el Complejo Turístico Baños del Inca, Cajamarca, 2002; Lima: Instituto Nacional de Cultura.

WATANABE, S. \& UGAZ, J., 2002 - Informe Preliminar del Proyecto de Investigación Arqueológica en el Complejo Turístico de Baños del Inca; Cajamarca-Lima: Instituto Nacional de Cultura.

WIENER, C., 1993 [1880] - Perú y Bolivia: Relato de Viaje, 859 pp.; Lima: Instituto Francés de Estudios Andinos, Universidad Nacional Mayor de San Marcos. 